\title{
Reducing Radiation Damage in Soft Matter with Femtosecond Timed Single-Electron Packets
}

\author{
Elisah J. VandenBussche and David J. Flannigan* \\ Department of Chemical Engineering and Materials Science, University of Minnesota, \\ 421 Washington Avenue SE, Minneapolis, MN 55455, USA
}

*Correspondence to: $\underline{\text { flan0076@umn.edu }},+1$ 612-625-3867

Abstract: Despite development of myriad mitigation methods, radiation damage continues to be a major limiting factor in transmission electron microscopy. Intriguing results have been reported using pulsed-laser driven and chopped electron beams for modulated dose delivery, but the underlying relationships and effects remain unclear. Indeed, delivering precisely-timed single-electron packets to the specimen has yet to be systematically explored, and no direct comparisons to conventional methods within a common parameter space have been made. Here, using a model linear saturated hydrocarbon ( $n$-hexatriacontane, $\mathrm{C}_{36} \mathrm{H}_{74}$ ), we show that preciselytimed delivery of each electron to the specimen, with a well-defined and uniform time between arrival, leads to a repeatable reduction in damage compared to conventional ultralow-dose methods for the same dose rate and the same accumulated dose. Using a femtosecond pulsed laser to confine the probability of electron emission to a 300-fs temporal window, we find damage to be sensitively dependent on the time between electron arrival (controlled with the laser repetition rate) and on the number of electrons per packet (controlled with the laser-pulse energy). Relative arrival times of 5, 20, and $100 \mu$ s were tested for electron packets comprised of, on average, 1, 5, and 20 electrons. In general, damage increased with decreasing time between electrons and, more substantially, with increasing electron number. Further, we find that improvements relative to conventional methods vanish once a threshold number of electrons per packet is reached. The results indicate that precise electron-by-electron dose delivery leads to a repeatable reduction in irreversible structural damage, and the systematic studies indicate this arises from control of the time between sequential electrons arriving within the same damage radius, all else being equal.

30 Keywords: radiation damage; electron microscopy; ultrafast electron microscopy 
In transmission electron microscopy (TEM), structural damage caused during electronspecimen interactions results in significant degradation of spatial resolution, which ultimately limits the quality and the accuracy of the information obtained. Indeed, the progression of damage during exposure leads to uncertainties in agreement between the acquired data and the intrinsic (i.e., artifact-free) properties of the material. The specific nature of the damage and the overall degree to which it occurs depends on a number of variables; including the electron-beam characteristics, the specimen properties, and the experimental requirements (1). As such, development of methods for minimizing deleterious effects of beam damage and for providing routes to elucidating the nature and the relative influence of fundamental mechanisms at work requires detailed, systematic exploration of a large, multivariable parameter space.

Though the factors and the mechanisms associated with beam damage are numerous and multi-faceted, a number of practical approaches have been shown to significantly reduce negative effects of radiolysis, the damage mechanism most relevant to radiation-sensitive organic matter (2). For example, cryogenic methods operate by holding the specimen at reduced temperatures during exposure in order to lower diffusion and kinetic reaction rates, to reduce mass loss, and to minimize the impact of beam-induced thermal effects, especially for poorlyconducting materials $(3,4)$. This has led to significant advances in the ability to study highlysensitive specimens, such as soft matter and biological structures, at sub-nanometer spatial scales (5-8). Radiolysis in particular can also be reduced by going to higher accelerating voltages owing to associated lower inelastic scattering cross sections, though a commensurate decrease in overall scattering signal also occurs (2). Minimizing exposure of the specimen to electron irradiation via low-dose and low-dose-rate methods and the use of conductive coatings and substrates have also proven beneficial (9-13). Further related to controlling damage through 
dose, the method of dose fractionation, which is based on the acquisition of fast, multi-frame scans or brief, stroboscopic multi-image series, has been shown to be a viable approach for minimizing beam-induced artifacts (14-16).

Transfer of energy from an incident electron to a material initiates a cascade of structural and electronic excitations, each of which is active within a characteristic temporal window (17). Accordingly, interesting questions can be posed pertaining to the feasibility of taking advantage of the temporal aspects of dose delivery in order to mitigate damage. Indeed, self-healing has been implicated in the recovery and the dose-rate-dependent damage thresholds observed for inorganic materials, biomolecules, and tissues (18-21). In light of this, delivering dose in a segmented fashion with a well-defined duration between each packet of electrons is a potentially intriguing approach to reducing or even circumventing the negative effects of certain damage mechanisms. Interestingly, the effects of pulsed-electron irradiation on damage to tissues, cells, and biomolecules is studied in the field of radiobiology, often by utilizing femtosecond (fs) pulsed-laser plasma accelerators $(21,22)$. In electron microscopy, the potential benefits of using a pulsed electron beam to mitigate damage have been speculated upon for decades (23-25). Further, though not attempting to leverage relaxation or self-healing, proposals for extending the concept of diffract-before-destroy to TEM have also been discussed (26-29). In this approach, sufficient information would be acquired before any damage has occurred by using a single, brief, large number-density electron packet. Unlike X-ray photons, however, electrons in such a dense packet will experience significant Coulomb repulsion, which will degrade coherence and limit image resolution $(30,31)$. This unwanted effect necessitates the use of high energies and/or post-emission recompression schemes (32-35). 
In order to systematically test the effects on damage of using a pulsed source in the TEM, and especially for direct comparisons to conventional methods, one ideally would have an extremely high level of control over key aspects of the discrete electron packets. Such aspects include the number of electrons in each packet, the duration of each packet, the time between each packet, and the number of packets per unit time. As will be described in more detail below, implementation of fs laser-driven photocathodes and picosecond chopped beams in TEMs provides such control $(24,25,36-40)$, and a few intriguing observations noting potential damage reduction when using a pulsed source have indeed been reported. For example, diffraction-spot intensities from crystals of copper 7,7,8,8-tetracyanoquinodimethane and polyethylene oxide under pulsed-beam illumination from a laser-driven photocathode were observed to be stable over inordinately long continuous exposure times $(41,42)$. More recently, apparent mitigation of damage was observed in $\mathrm{MgCl}_{2}$ when using a segmented beam consisting of picosecond electron bunches, each regularly spaced in time by $\sim 160$ ps (i.e., a repetition rate of $6 \mathrm{GHz}$ ) (43). However, as in pulsed-beam radiobiology studies (44), the precise effects on damage of pulsing the beam, and the relationship with dose rate, are largely unknown. Indeed, no study has yet determined the fundamental effects of precisely delivering each individual electron to the specimen with an extremely well-defined and highly-tunable time between each. Further, under such finely-controlled conditions, no direct comparisons have been made to conventional lowdose methods for precisely the same dose rate and the same total dose.

Here, we report our observations of a repeatable reduction in damage when using pulsed electron packets generated via fs laser excitation of a photocathode in a modified TEM as compared to a conventional thermionic beam for precisely the same dose rate and accumulated dose. By using fs laser excitation to precisely control the electron-emission process, we confine 
the probability of emission to a temporal range of $300 \mathrm{fs}$ (full-width at half-maximum, FWHM) for extremely regular peak-to-peak emission times. Using a Bragg-spot fading-curve method and a model beam-sensitive soft-matter material ( $n$-hexatriacontane, $\mathrm{C}_{36} \mathrm{H}_{74}, \mathrm{C} 36$ hereafter), we measure a nearly two-fold reduction in damage when the dose is delivered one electron at a time with up to $100 \mu$ s between the arrival of each at the specimen. That is, for thermionic beams of the same dose rate $\left[\right.$ e.g., as low as $7.8 \times 10^{-7} \mathrm{e} \cdot \AA^{-2} \cdot \mathrm{s}^{-1} ; 1.6$ femtoamp (fA) beam current; 0.01 zeptoamps per $\left.\mathrm{nm}^{2}\right]$, the observed damage is nearly double that of the pulsed beam for a common total dose. We also explore the effects of varying the duration between the arrival of each individual electron at the specimen and the number of electrons in each individual packet. In general, we find that reducing the duration between electron arrival or increasing the number of electrons in each packet leads to an increase in damage to the point where the effects of pulsing the beam are indistinguishable from conventional approaches, indicative of a threshold effect.

\section{Results and Discussion}

Pulsed-Beam Method. An overview of the concept and approach is provided in Fig. 1. The key advance leveraged here is the use of a fs laser to control electron emission from the TEM source (see Fig. S1 in the Supporting Information). In this way, one can dictate precisely when emission occurs, how often it occurs, and how many electrons are emitted during each pulse. Here, emission is confined to a temporal window as small as 300 fs FWHM (laser pulse duration), is restricted to occurring as infrequently as once every $100 \mu$ s (laser repetition rate, $f$ ), and is limited to as few as one electron per pulse [laser pulse energy; see Fig. S2]. As a result, a precisely-defined, extremely-regular time between electron arrival can be applied by varying $f$. 
Regularity arises from the temporal emission probability $[P(t)]$ being confined to the laser-pulse duration; in pulsed mode, emission occurs only when photons are incident on the photocathode source [i.e., $P(t)=0$ between laser pulses]. As such, sequential electron emission is restricted to occurring within a temporal window defined as $f^{1} \pm 150$ fs for a train of 300 -fs laser pulses (for negligible laser pulse-to-pulse jitter). Note that subsequent electron acceleration and mismatches in photon energy and cathode work function will add an effective timing jitter approximately on the order of the laser-pulse duration (45). During a particular experiment, shot-to-shot pulseenergy stability and statistics of photoemission dictate the number of electrons emitted during each pulse. Here, the stability was such that an error of approximately $\pm 10 \%$ (one standard deviation from the mean) was measured for one electron per packet, while it was substantially smaller for 5 and 20 electrons per packet (see Fig. S2 and Table S1). Note that the value of $P(t)$ for conventional thermionic emission is both non-zero and constant during the entire exposure time (i.e., emission probability is the same throughout). It is also important to note that longterm stability of the pulsed photoelectron beam was high, varying for example by $\pm 0.3 \%$ over a 24-hour period during a successful experiment (see Fig. S3). Also note that all experiments were performed at room temperature; because the objective was to test the hypothesis that - all else being equal - using a strictly-controlled pulsed electron beam can lead to a reduction in specimen damage, conducting the experiments at cryogenic temperatures was not necessary and, additionally, would add an unnecessary level of experimental complexity. 


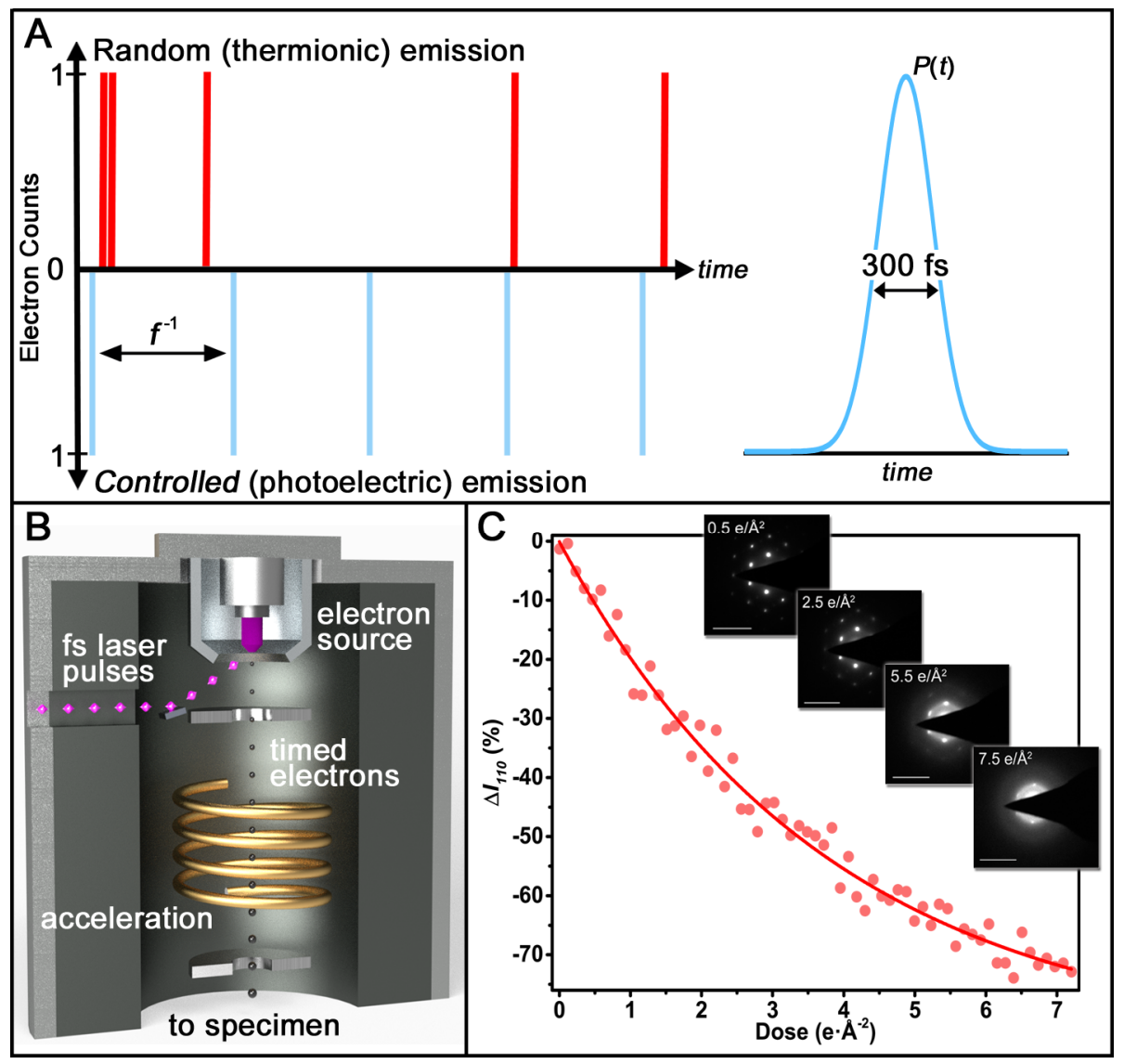

Fig. 1. Experimental approach. (A) Concept of controlled, precisely-timed electron emission compared to conventional random (thermionic) emission for the same beam current. The laser repetition rate $\left(f\right.$; pulses per second) dictates the time between electron emission $\left(f^{1}\right)$ for the pulsed approach. Laser-pulse energy is tuned to select the desired number of electrons, and the temporal emission probability $[P(t)]$ is confined to the pulse duration (here, $300 \mathrm{fs}$ FWHM). (B) Schematic of the source region of the modified TEM. The fs laser pulses enter through an optical port and are directed onto the source to generate timed electron packets. These are then accelerated and directed to the specimen using conventional methods. (C) Example of the 10 Bragg-spot fading-curve method used to monitor damage [see also Fig. S4 and the discussion in the Supporting Information] for a large total dose relative to the pulsed- and random-beam radiation-damage experiments that were the focus of study. Shown is representative data of the 
fading of 110 Bragg spots from a C36 microcrystal during random (thermionic) irradiation. Here, $\Delta I_{110}=\left(I_{110}-I_{0,110}\right) / I_{0}, 110$, where $I_{110}$ is the average intensity of the 110 spots at a specific dose $\left(\mathrm{e} \cdot \AA^{-2}\right)$, and $I_{0,110}$ is the initial average intensity at nominally zero dose.

One Electron Per Packet, 100 us Between Each Electron. Effects of pulsed- and random thermionic-beam irradiation on 110 Bragg-spot intensities from individual C36 microcrystals for an identical ultralow dose rate of $7.8 \times 10^{-7} \mathrm{e} \cdot \AA^{-2} \cdot \mathrm{s}^{-1}$ are summarized in Fig. 2 (see the Supporting Information for a description of the specimen preparation). For the pulsed mode, the number of electrons generated per laser pulse was $1.03 \pm 0.15$, and the time between arrival of each at the C36 microcrystal was $f^{1}=100 \mu$ s (see the Supporting Information for details on the measurement of the number of electrons per packet). Intensities were monitored up to an accumulated dose of $0.066 \mathrm{e} \cdot \AA^{-2}$ (see also Fig. S5). Linear intensity decreases were observed for each over this relatively low range of accumulated dose. When elastic-bending and dynamicalscattering effects are negligible, Bragg-spot intensity attenuation as a function of dose is indicative of a loss of crystallinity due to increasing disorder and irreversible structural damage (46). Here, the slopes of the responses differ by a factor of 1.8, with the rate of damage sustained for the pulsed beam being lower than that of the thermionic beam despite being applied at the same dose rate. See Figs. S6 and S7 and the Supporting Information for descriptions of the methods used to measure beam current and beam size. Note that care was taken to ensure the C36 microcrystals studied were the same thickness (see Fig. S8 and the Supporting Information). Note also that, despite the necessary long experiment times, stability of the lab environment and the instruments was such that negligible net specimen drift occurred (i.e., drift occurred about a single center-of-mass position after system and lab equilibration). This resulted in individual 
C36 microcrystals remaining positioned under the beam for the entire duration of each experiment for both thermionic and pulsed-beam measurements (i.e., net specimen drift was negligible for both conditions, as expected owing to the same dose rates and accumulated doses). This controls for systematic errors arising from potentially different net specimen drifts for the pulsed vs. thermionic experiments. Further, owing to the results, net specimen drift would need to consistently be greater for the thermionic condition despite being administered at the same dose rates (beam current and beam size) and under the same lab- and instrument-stability requirements as the pulsed condition. See Fig. S9 and the Supporting Information for an illustrative example of the typical specimen spatial stability achieved and for a discussion of how lab-environment and instrument stabilities were monitored and assessed.
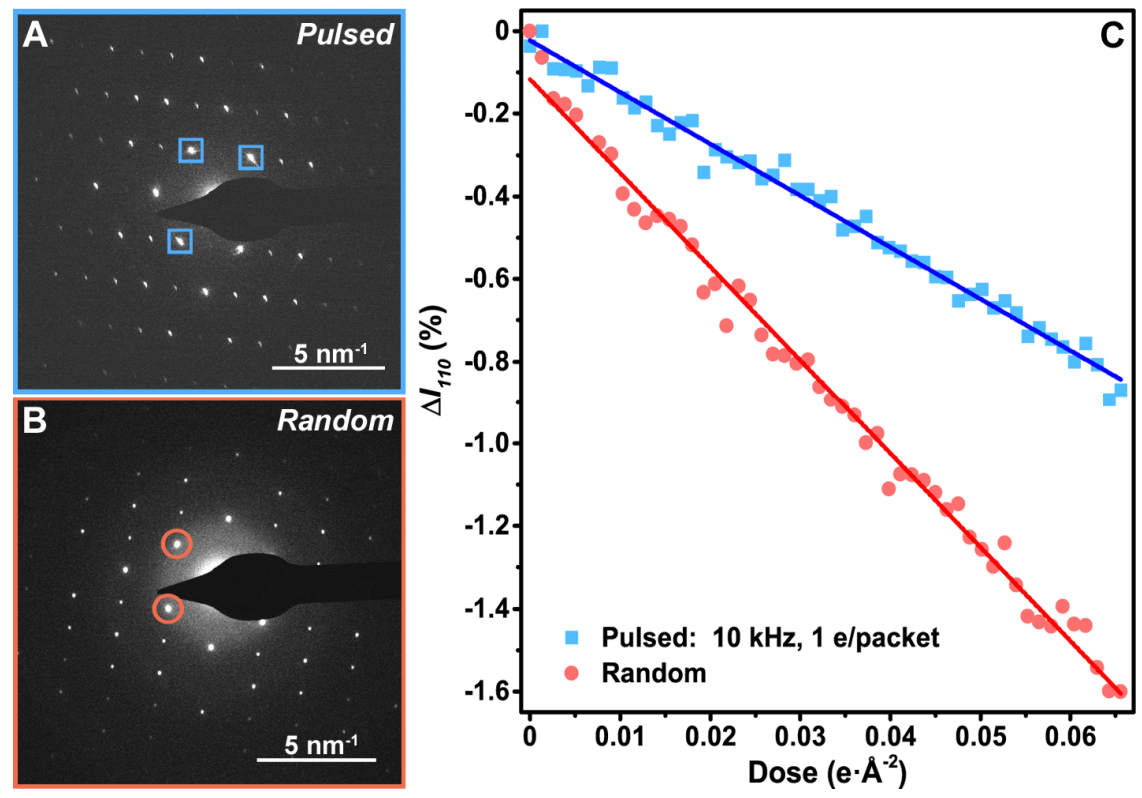

Fig. 2. Comparison of pulsed and random (thermionic) electron-beam damage for the same dose rate $\left(7.8 \times 10^{-7} \mathrm{e} \cdot \AA^{-2} \cdot \mathrm{s}^{-1}\right)$. Representative (A) pulsed- and (B) random-beam diffraction patterns of a C36 microcrystal with the 110 Bragg spots used to generate the fading curves in panel (C) highlighted. (C) Fading curves and least-squares fits for the pulsed and random beams. The 
slope of the line fit to the random-beam data is steeper than that of the pulsed beam by a factor of 1.8. Pertinent specifications are as follows: Pulsed-beam current and illuminated area were 1.6 $\pm 0.24 \mathrm{fA}$ and $132.4 \mu \mathrm{m}^{2}$, respectively $\left(0.01\right.$ zeptoamps per $\left.\mathrm{nm}^{2}\right)$. Random-beam current and illuminated area were $1.6 \pm 0.22 \mathrm{fA}$ and $131.5 \mu \mathrm{m}^{2}$, respectively. The pulsed beam was generated with a 300-fs FWHM pulsed laser operated at $f=10 \mathrm{kHz}\left(f^{1}=100 \mu \mathrm{s}\right)$. The laser pulse energy incident on the TEM photocathode was set such that the number of electrons per packet was measured to be $1.03 \pm 0.15$.

Because the only difference between the two beam types was the temporal profile of electron arrivals (i.e., electrons may arrive more closely together in time for conventional thermionic emission, whereas this is strictly controlled in the pulsed-beam case), the results shown in Fig. 2 suggest that damage caused to the specimen is sensitive to shorter-than-average durations between electron arrivals. This then raises questions pertaining to the relationship between sequential electron arrival and damage accumulation in the specimen. That is, if two electrons arrive within a particular time interval and spatial proximity to one another, a description must be formed regarding what additional damage this would cause compared to the case in which the electrons arrive $100 \mu$ s apart, as well as what the relevant spatial radius and time scales are for additional damage to occur. To explore this, insight is drawn from the relevant physical damage mechanisms.

The primary damage mechanism - excitation and ejection of specimen electrons caused by collisions with incident electrons - occurs in femtoseconds and is essentially confined to a single molecule (47-49). This is because the inelastic scattering cross section of a high-energy electron makes it unlikely that more than one collision per incident electron occurs. Secondary 
electron damage caused by ejected and Auger electrons, which has been estimated to cause up to $80 \%$ of the damage to the specimen (50), also occurs very quickly, over tens of femtoseconds. Because the energy deposited by incident electrons (37 eV, on average, for paraffins) is much higher than the threshold for damage $(4.8 \mathrm{eV})$, a single secondary electron can break multiple bonds and excite multiple additional electrons before its energy drops below the damage threshold (50). This in essence constitutes a non-linear relationship between the number of damage events and the number of incident inelastic collisions. While the damage radius of an individual event for secondary electrons is spatially localized - on the order of ten unit cells due to the relatively large scattering cross sections (51-53), the non-trivial probability of multiple events occurring from one electron leads to an increased chance of multiple radicals and additional excited electrons being formed. Therefore, the damage radius associated with secondary-electron excitation is significantly larger when relevant aspects of all associated excitations are accounted for. That is, the damage radius for all associated events arising from a single incident inelastic collision includes typical diffusion lengths, mean free paths, and lifetimes for the radicals generated, the products of the radical reactions, and the phonons excited during relaxation of each event.

Occurring on the order of nanoseconds after initial formation, free radicals react with the surrounding lattice or with small, diffusing molecules (e.g., free $\mathrm{H}_{2}$ and light hydrocarbons) (49), though several factors dictate the precise reaction rate; including molecular identity, free-radical site reactivity and geometry, radical concentration, and local lattice energy. Degradation of unstable species follows the initial reactions, subsequently causing structural deterioration manifest as loss of crystallinity and Bragg-spot intensity fading $(49,54)$. This process continues for hundreds of nanoseconds to microseconds, though again the precise duration is dependent 
upon free-radical concentration and on local energy $(49,55)$. Because the manner in which this process proceeds is dependent upon local energy, radical concentration, and diffusion rates, the thermal energy available significantly influences the damage sustained. For example, a temperature increase of $1 \mathrm{~K}$ produces an additional 3\% damage, while an increase of $9 \mathrm{~K}$ produces an additional $28 \%(17,56)$. This is noteworthy because while the global increase in equilibrium temperature is negligible at low beam currents $(2,17,57,58)$, the energy released locally during excited electron relaxation is not, as evidenced by the processes described above. Illustratively, excited phonons vibrating in the 5 to $15 \mathrm{THz}$ range have 3 to $10 \mathrm{meV}$ of energy, which corresponds to local temperature increase of 30 to $110 \mathrm{~K}(59,60)$. (Note that while it may not be technically correct to use temperature as a descriptor of thermal energy at these length scales, it is nonetheless instructive to consider the $k T$ energies involved.) This energy will be distributed over tens to hundreds of nanometers, per the damage radius discussion above, and will persist for hundreds of nanoseconds to microseconds $(61,62)$.

From this discussion, it is therefore reasonable to assume that local phonon excitation, while not sufficient to significantly raise the global thermalized lattice temperature, can cause appreciable increases in energy locally at the excitation site, thereby causing a non-linear increase in the damage caused by free radicals and higher diffusion rates. The combination of dominant damage mechanisms, and the associated compounding synergistic effects (e.g., Arrhenius-type increases in reaction and diffusion rates with local energy), suggests that incident electrons impinging upon the specimen within hundreds of nanoseconds to microseconds and tens to hundreds of nanometers of one another could produce a degree of irreversible damage that would not occur if reactive species, phonons, and secondary electrons were allowed significantly more relaxation time between electron arrival. Following these arguments, one 
would then expect events of electrons arriving with shorter-than-average durations between them to result in more extensive irreversible structural damage from otherwise reversible processes owing to increased accumulation of local lattice energy and a higher spatiotemporal number density (i.e., species per volume per time) of reactive species during moments of greater instantaneous dose in the random beam. See the Supporting Information for details of the measures taken to account and control for sources of both random and systematic error.

One Electron Per Packet, 5 us Between Each Electron. To test the above-stated hypotheses, a set of pulsed-beam experiments were conducted, wherein both the time between electrons $\left(f^{1}\right)$ and the number of electrons in each packet were systematically varied and compared to the random thermionic-beam method and to one another. Figure 3 shows the result of reducing $f^{1}$ to $5 \mu \mathrm{s}(f=200 \mathrm{kHz})$ for $1.04 \pm 0.11$ electrons per packet, again compared to the random-beam approach administered at the same dose rate (in this case, $9 \times 10^{-6} \mathrm{e} \cdot \AA^{-2} \cdot \mathrm{s}^{-1}$ owing to a higher $f$ ). A statistically-significant reduction in damage at a given accumulated dose (here, up to 0.077 e. $\AA^{-2}$ ) is again observed for the pulsed beam. Interestingly, damage for the pulsed-beam approach is again reduced by nearly a factor of two relative to the conventional low-dose method. This reduction in damage recurred nearly identically in a repetition of the experiment (see Table S1), demonstrating the repeatability of the result. The damage at a common accumulated dose (e.g., $\left.0.066 \mathrm{e} \cdot \AA^{-2}\right)$, however, is higher by approximately $15 \%$ for both the pulsed and the random beams when compared to that sustained with the lower dose rate shown in Fig. 2. This supports the hypothesis that contributions by irreversible aspects of otherwise reversible mechanisms to the accumulated damage sensitively depend upon the time between arrival of each individual electron at the specimen. 


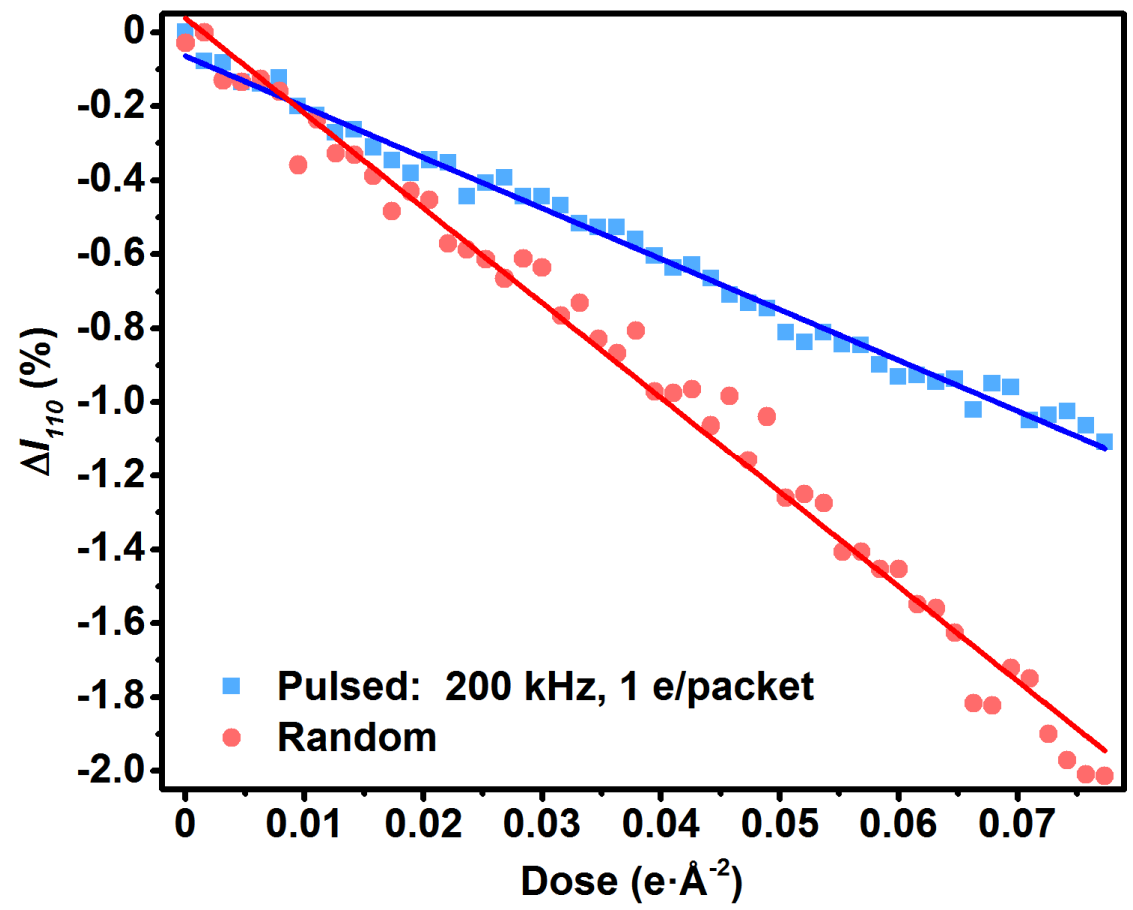

Fig. 3. Comparison of damage induced by pulsed and random (thermionic) electron-beam irradiation for the same dose rate $\left(9 \times 10^{-6} \mathrm{e} \cdot \AA^{-2} \cdot \mathrm{s}^{-1}\right)$. Fading curves and least-squares fits for the pulsed and random beams are shown. The slope of the line fit to the random-beam data is steeper than that of the pulsed beam by a factor of 1.9. Pertinent specifications are as follows: Pulsed-beam current and illuminated area were $31.7 \pm 3.54 \mathrm{fA}$ and $219.4 \mu \mathrm{m}^{2}$, respectively. Random-beam current and illuminated area were $32.0 \pm 3.45 \mathrm{fA}$ and $220.7 \mu \mathrm{m}^{2}$, respectively. The pulsed beam was generated with a 300-fs FWHM pulsed laser operated at $f=200 \mathrm{kHz}\left(f^{1}=\right.$ $5 \mu \mathrm{s})$. The laser pulse energy was set such that the number of electrons per packet was measured $10 \quad$ to be $1.04 \pm 0.11$.

Increasing the Number of Electrons Per Packet. The effect of the number of electrons in each packet on damage was also studied, the results of which are summarized in Fig. 4. Because two factors were tested $\left(f^{1}\right.$ and the number of electrons per packet), a two-level factorial approach was taken in order to avoid conflation. It was observed that the degree of damage sustained at a 
given accumulated dose is significantly higher when the number of electrons per packet is increased (here, from one to five; Fig. 4A). Reducing $f^{1}$ for the 5-electron packets from $100 \mu \mathrm{s}$ to $5 \mu$ s produced a further increase in damage, potentially to a larger degree than for the 1electron packets. An analysis of variance shows that both factors are statistically-significant predictors of damage (to 95\% confidence), but no super-additive effect was apparent at this confidence level. That is, the results do not indicate that varying both factors produces any additional effect beyond the additive ones observed. This said, an unexplored region of the verylarge parameter space may indeed exist where super-additive effects do occur. Interestingly, the deleterious effects caused by increasing the number of electrons per packet are substantially more significant than those caused by decreasing $f^{1}$; the five-fold increase in electrons per packet produces a greater increase in damage than a five-fold decrease in $f^{1}$.

The dramatic increase in damage caused by a modest increase in the number of electrons per packet suggests that, for a given $f^{1}$, a point should be reached where all benefits provided by pulsing the source compared to the conventional low-dose approach are lost. Figure 4B shows a comparison of pulsed- and random-beam irradiation, where the number of electrons per packet for the pulsed beam was increased to $20\left(f^{1}=5 \mu \mathrm{s}\right.$; same dose rate $\left.=1.8 \times 10^{-4} \mathrm{e} \cdot \AA^{-2} \cdot \mathrm{s}^{-1}\right)$. As can be seen, no statistical difference (to 95\% confidence) at a given dose (here, $0.063 \mathrm{e} \cdot \AA^{-2}$ ) is present, indicating that the amount of damage caused by the arrival of 20 electrons at the specimen over an estimated 2-ps time period [increased from 300 fs due to Coulomb repulsion (63)] is comparable to that occurring during the conventional low-dose approach. It is reasonable to speculate that the quantitative behaviors observed here are material dependent, but more work is needed. Taken together, these results support the hypothesis that, despite being administered at the same dose rate, the statistical generation of multiple electrons over a shorter- 
than-average period of time during conventional emission processes is the source of the observed increased damage compared to the precision pulsed-source approach.
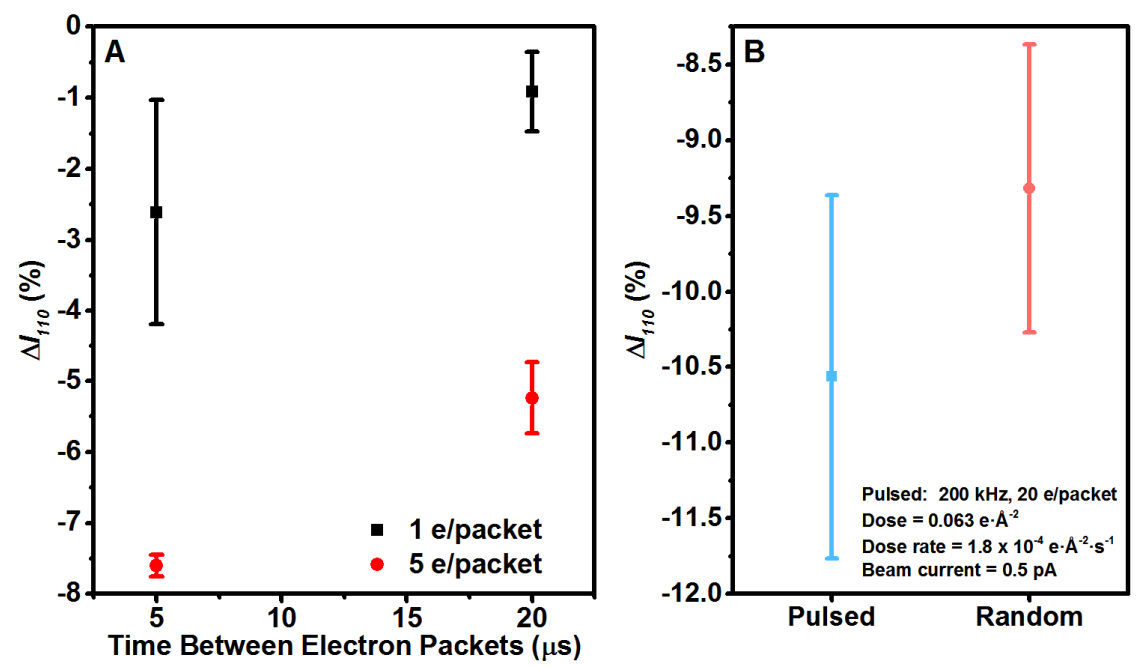

Fig. 4. Effect of the number of electrons per packet and the duration between each packet on damage. (A) Change in $I_{110}$ after a total accumulated dose of $0.063 \mathrm{e} \cdot \AA^{-2}$. Error bars represent the standard error over four separate experiments. All data points were generated using a pulsed beam, and the same size specimen area was irradiated for each (within 0.5\%). (B) Change in $I_{110}$ after a total accumulated dose of $0.063 \mathrm{e} \cdot \AA^{-2}$ for a pulsed beam with 20 electrons per packet and $f^{1}=5 \mu \mathrm{s}(f=200 \mathrm{kHz})$ compared to a random (thermionic) beam of the same current $(0.5 \mathrm{pA})$ and for the same size irradiated specimen area (within $1 \%$; same dose rate $=1.8 \times 10^{-4} \mathrm{e} \cdot \AA^{-2} \cdot \mathrm{s}^{-1}$ ). Error bars represent the standard error over five separate experiments.

\section{Summary and Conclusions}

Via systematic studies and direct, statistical comparisons to conventional methods, we have conclusively shown that delivering dose using a precise, highly-controlled pulsed-beam approach in a TEM can reduce the damage sustained by beam-sensitive materials. We 
accomplish this by using the highly regular and stable emission from a fs pulsed laser to strictly control critical aspects of electron emission from the source. We find that the degree of damage is sensitive to both the duration between arrival of each electron at the specimen and to the number of electrons per packet. Increased damage with increased electrons per packet, as well as the observation of a loss of advantageous effects, suggests the temporally random emission occurring during conventional low-dose methods is a significant contributor to beam-induced damage and that the pulsed-beam approach in essence mitigates the cumulative effects of sequential inelastic events occurring within a damage-mechanism-specific spatiotemporal window. The results of this work open an entirely new avenue of research into fundamental studies of radiation-damage mechanisms and into mitigation of the associated deleterious effects. Myriad future studies are envisioned, including quantifiably testing the effects on spatial resolution and extension of the approach to probing biological structures, biomaterials, biological macromolecules, radiation-sensitive organic and inorganic materials (e.g., organic semiconductors, hybrid perovskites, and zeolites), liquid environments, and cryo-EM.

\section{References:}

1. Egerton RF (2019) Radiation damage to organic and inorganic specimens in the TEM. Micron 119:72-87.

2. Egerton RF (2013) Control of radiation damage in the TEM. Ultramicroscopy 127:100108.

3. Glaeser RM, Taylor KA (1978) Radiation damage relative to transmission electron microscopy of biological specimens at low temperature: A review. J Microsc (Oxford U K) 112:127-138. 
4. Dubochet J (2018) On the development of electron cryo-microscopy (Nobel Lecture). Angew Chem Int Ed 57:10842-10846.

5. Frank J (2009) Single-particle reconstruction of biological macromolecules in electron microscopy - 30 years. $Q$ Rev Biophys 42:139-158.

6. Kourkoutis LF, Plitzko JM, Baumeister W (2012) Electron microscopy of biological materials at the nanometer scale. Апnu Rev Mater Res 42:33-58.

7. Cheng Y (2018) Single-particle cryo-EM-How did it get here and where will it go. Science 361:876-880.

8. Henderson R (2018) From electron crystallography to single particle cryoEM (Nobel Lecture). Angew Chem Int Ed 57:10804-10825.

9. Salih SM, Cosslett VE (1974) Reduction in electron-irradiation damage to organic compounds by conducting coatings. Philos Mag 30:225-228.

10. Unwin PNT, Henderson R (1975) Molecular structure determination by electron microscopy of unstained crystalline specimens. J Mol Biol 94:425-440.

11. Fryer JR (1984) Radiation damage in organic crystalline films. Ultramicroscopy 14:227236.

12. Evans JE, Hetherington C, Kirkland A, Chang LY, Stahlberg H, Browning N (2008) Low-dose aberration corrected cryo-electron microscopy of organic specimens. Ultramicroscopy 108:1636-1644.

13. Keskin S, de Jonge N (2018) Reduced radiation damage in transmission electron microscopy of proteins in graphene liquid cells. Nano Lett 18:7435-7440. 
14. Glaeser RM, McMullan G, Faruqi AR, Henderson R (2011) Images of paraffin monolayer crystals with perfect contrast: Minimization of beam-induced specimen motion. Ultramicroscopy 111:90-100.

15. Li X, Mooney P, Zheng S, Booth CR, Braunfeld MB, Gubbens S, Agard DA, Cheng Y (2013) Electron counting and beam-induced motion correction enable near-atomicresolution single-particle cryo-EM. Nat Methods 10:584-590.

16. Zhang D, Zhu Y, Liu L, Ying X, Hsiung C-E, Sougrat R, Li K, Han Y (2018) Atomicresolution transmission electron microscopy of electron beam-sensitive crystalline materials. Science 359:675-679.

17. Grubb DT (1974) Radiation damage and electron microscopy of organic polymers. J Mater Sci 9:1715-1736.

18. Jiang N, Spence JCH (2012) On the dose-rate threshold of beam damage in TEM. Ultramicroscopy 113:77-82.

19. Favaudon V, Caplier L, Monceau V, Pouzoulet F, Sayarath M, Fouillade C, Poupon M-F, Brito I, Hupé P, Bourhis J, Hall J, Fontaine JJ, Vozenin MC (2014) Ultrahigh dose-rate FLASH irradiation increases the differential response between normal and tumor tissue in mice. Sci Transl Med 6:245ra293.

20. Johnston-Peck AC, DuChene JS, Roberts AD, Wei WD, Herzing AA (2016) Dose-ratedependent damage of cerium dioxide in the scanning transmission electron microscope. Ultramicroscopy 170:1-9.

21. Babayan N, Hovhannisyan G, Grigoryan B, Grigoryan R, Sarkisyan N, Tsakanova G, Haroutiunian S, Aroutiounian R (2017) Dose-rate effect of ultrashort electron beam radiation on DNA damage and repair in vitro. J Radiat Res 58:894-897. 
22. Laschinsky L, Baumann M, Beyreuther E, Enghardt W, Kaluza M, Karsch L, Lessmann E, Naumburger D, Nicolai M, Richter C, Sauerbrey R, Schlenvoigt H-P, Pawelke J (2012) Radiobiological effectiveness of laser accelerated electrons in comparison to electron beams from a conventional linear accelerator. $J$ Radiat Res 53:395-403.

23. Bonham RA, Kennerly RE (1978) Pulsed electron beams to reduce radiation damage? Ann N Y Acad Sci 306:85-94.

24. King WE, Campbell GH, Frank A, Reed B, Schmerge JF, Siwick BJ, Stuart BC, Weber PM (2005) Ultrafast electron microscopy in materials science, biology, and chemistry. $J$ Appl Phys 97:111101.

25. Lobastov VA, Srinivasan R, Zewail AH (2005) Four-dimensional ultrafast electron microscopy. Proc Natl Acad Sci USA 102:7069-7073.

26. Chapman HN, Caleman C, Timneanu N (2014) Diffraction before destruction. Philos Trans $R$ Soc B 369:20130313.

27. Egerton RF (2015) Outrun radiation damage with electrons? Adv Struct Chem Imaging $1: 5$

28. Spence JCH, Subramanian G, Musumeci P (2015) Hollow cone illumination for fast TEM, and outrunning damage with electrons. J Phys B At Mol Opt Phys 48:214003.

29. Spence JCH (2017) Outrunning damage: Electrons vs X-rays - timescales and mechanisms. Struct Dyn 4:044027.

30. Armstrong MR, Boyden K, Browning ND, Campbell GH, Colvin JD, DeHope WJ, Frank AM, Gibson DJ, Hartemann F, Kim JS, King WE, LaGrange TB, Pyke BJ, Reed BW, Shuttlesworth RM, Stuart BC, Torralva BR (2007) Practical considerations for high 
spatial and temporal resolution dynamic transmission electron microscopy.

Ultramicroscopy 107:356-367.

31. Gahlmann A, Park ST, Zewail AH (2008) Ultrashort electron pulses for diffraction, crystallography and microscopy: Theoretical and experimental resolutions. Phys Chem Chem Phys 10:2894-2909.

32. Li RK, Musumeci P (2014) Single-shot MeV transmission electron microscopy with picosecond temporal resolution. Phys Rev Appl 2:024003.

33. Weathersby SP, Brown G, Centurion M, Chase TF, Coffee R, Corbett J, Eichner JP, Frisch JC, Fry AR, Gühr M, Hartmann N, Hast C, Hettel R, Jobe RK, Jongewaard EN, Lewandowski JR, Li RK, Lindenberg AM, Makasyuk I, May JE, McCormick D, Nguyen MN, Reid AH, Shen X, Sokolowski-Tinten K, Vecchione T, Vetter SL, Wu J, Yang J, Dürr HA, Wang XJ (2015) Mega-electron-volt ultrafast electron diffraction at SLAC National Accelerator Laboratory. Rev Sci Instrum 86:073702.

34. Kealhofer C, Schneider W, Ehberger D, Ryabov A, Krausz F, Baum P (2016) All-optical control and metrology of electron pulses. Science 352:429-433.

35. Mankos M, Shadman K, Siwick BJ (2017) A novel electron mirror pulse compressor. Ultramicroscopy 183:77-83.

36. Qiu JQ, Ha GH, Jing CG, Baryshev SV, Reed BW, Lau JW, Zhu YM (2016) GHz laserfree time-resolved transmission electron microscopy: A stroboscopic high-duty-cycle method. Ultramicroscopy 161:130-136.

37. Verhoeven W, van Rens JFM, Kieft ER, Mutsaers PHA, Luiten OJ (2018) High quality ultrafast transmission electron microscopy using resonant microwave cavities. Ultramicroscopy 188:85-89. 
38. Bormann R, Strauch S, Schäfer S, Roper C (2015) An ultrafast electron microscope gun driven by two-photon photoemission from a nanotip cathode. J Appl Phys 118:173105.

39. Feist A, Bach N, Rubiano da Silva N, Danz T, Möller M, Priebe KE, Domröse T, Gatzmann JG, Rost S, Schauss J, Strauch S, Bormann R, Sivis M, Schäfer S, Roper C (2017) Ultrafast transmission electron microscopy using a laser-driven field emitter: Femtosecond resolution with a high coherence electron beam. Ultramicroscopy 176:6373.

40. Houdellier F, Caruso GM, Weber S, Kociak M, Arbouet A (2018) Development of a high brightness ultrafast transmission electron microscope based on a laser-driven cold field emission source. Ultramicroscopy 186:128-138.

41. Flannigan DJ, Lobastov VA, Zewail AH (2007) Controlled nanoscale mechanical phenomena discovered with ultrafast electron microscopy. Angew Chem Int Ed 46:92069210.

42. Kwon O-H, Ortalan V, Zewail AH (2011) Macromolecular structural dynamics visualized by pulsed dose control in 4D electron microscopy. Proc Natl Acad Sci USA 108:6026-6031.

43. Kisielowski C, Specht P, Freitag B, Kieft ER, Verhoeven W, van Rens JFM, Mutsaers P, Luiten J, Rozeveld S, Kang J, McKenna AJ, Nickias P, Yancey DF (2019) Discovering hidden material properties of $\mathrm{MgCl}_{2}$ at atomic resolution with structured temporal electron illumination of picosecond time resolution. Adv Funct Mater 29:1807818.

44. Laschinsky L, Karsch L, Leßmann E, Oppelt M, Pawelke J, Richter C, Schürer M, Beyreuther E (2016) Radiobiological influence of megavoltage electron pulses of ultrahigh pulse dose rate on normal tissue cells. Radiat Environ Biophys 55:381-391. 
45. Aidelsburger M, Kirchner FO, Krausz F, Baum P (2010) Single-electron pulses for ultrafast diffraction. Proc Natl Acad Sci USA 107:19714-19719.

46. Dorset D (1980) Electron diffraction intensities from bent molecular organic crystals. Acta Crystallogr Sect A 36:592-600.

47. Dewhurst HA, Samuel AH, Magee JL (1954) A theoretical study of the radiation chemistry of water and aqueous solutions. Radiat Res 1:62-84.

48. Fryer JR, Holland F (1984) High resolution electron microscopy of molecular crystals III. Radiation processes at room temperature. Proc R Soc A 393:353-369.

49. Fryer JR (1987) The effect of dose rate on imaging aromatic organic crystals. Ultramicroscopy 23:321-327.

50. Egerton RF (2012) Mechanisms of radiation damage in beam-sensitive specimens, for TEM accelerating voltages between 10 and $300 \mathrm{kV}$. Microsc Res Tech 75:1550-1556.

51. Williams DB, Carter CB (2009) Transmission Electron Microscopy: A Textbook for Materials Science. Springer: New York.

52. Ashley JC, Williams MW (1980) Electron mean free paths in solid organic insulators. Radiat Res 81:364-373.

53. Gauduel Y, Friztler S, Hallou A, Glinec Y, Malka V (2004) Femtosecond relativistic electron beam triggered early bioradical events. Proc SPIE 5463:86-96.

54. Henderson R, Glaeser RM (1985) Quantitative analysis of image contrast in electron micrographs of beam sensitive materials. Ultramicroscopy 16:139-150.

55. Ungar G, Grubb DT, Keller A (1977) Spatial nonuniformity of cross-linking in crystalline alkanes of different chain length. Radiat Phys Chem 22:849-860. 
56. Orth VH, Fischer EW (1965) Änderungen der gitterstruktur hochpolymerer einkristalle durch bestrahlung im elektronenmikroskop. Die Makromolekulare Chemie 88:188-214.

57. Dorset DL, Turner JN (1976) Thermal effects in electron beam damage of organic crystals. Naturwissenschaften 63:145-146.

58. Egerton RF, Rauf I (1999) Dose-rate dependence of electron-induced mass loss from organic specimens. Ultramicroscopy 80:247-254.

59. Henry A, Chen G, Plimpton SJ, Thompson A (2010) 1D-to-3D transition of phonon heat conduction in polyethylene using molecular dynamics simulations. Phys Rev B 82:144308.

60. Wang X, Kaviany M, Huang B (2017) Phonon coupling and transport in individual polyethylene chains: A comparison study with the bulk crystal. Nanoscale 9:1802218031.

61. Cremons DR, Du DX, Flannigan DJ (2017) Picosecond phase-velocity dispersion of hypersonic phonons imaged with ultrafast electron microscopy. Phys Rev Mater 1:073801.

62. McKenna AJ, Eliason JK, Flannigan DJ (2017) Spatiotemporal evolution of coherent elastic strain waves in a single $\mathrm{MoS}_{2}$ flake. Nano Lett 17:3952-3958.

63. Plemmons DA, Flannigan DJ (2017) Ultrafast electron microscopy: Instrument response from the single-electron to high bunch-charge regimes. Chem Phys Lett 683:186-192.

Acknowledgments: We thank Alexander VandenBussche for assistance with making Figure 1. Funding: This material is based on work supported by the U.S. Department of Energy, Office of Science, Office of Basic Energy Sciences under Award No. DE-SC0018204. This material is 
based upon work partially supported by the National Science Foundation Graduate Research Fellowship Program under Grant No. DGE-1348264. Any opinions, findings, and conclusions or recommendations expressed in this material are those of the author(s) and do not necessarily reflect the views of the National Science Foundation. Acknowledgment is made to the Donors of the American Chemical Society Petroleum Research Fund for partial support of this research. Author contributions: VandenBussche's contributions were formal analysis, investigation, methodology, software, validation, visualization, writing - original draft, and writing - review and editing. Flannigan's contributions were conceptualization, formal analysis, funding acquisition, methodology, project administration, resources, supervision, visualization, writing original draft, and writing - review and editing. Competing interests: Authors declare no competing interests. Data and materials availability: All data is available in the main text or the supplementary materials. Data is available upon reasonable request from the corresponding author.

\section{Supporting Information:}

15 Materials and Methods

Figures S1-S9

Table S1 


\title{
Supporting Information for
}

\author{
Reducing Radiation Damage in Soft Matter with \\ Femtosecond Timed Single-Electron Packets
}

\author{
Elisah J. VandenBussche and David J. Flannigan \\ Correspondence to: flan0076@umn.edu
}

This file includes:

Materials and Methods

Figs. S1-S9

Table S1

\section{Materials and Methods}

\section{$\underline{\text { TEM and Laser }}$}

All experiments (thermionic and pulsed photoelectron) were conducted with a $200 \mathrm{kV}$ FEI Tecnai FEMTO transmission electron microscope (Thermo Fisher Scientific) equipped with a Gatan OneView CMOS camera. The Tecnai FEMTO is equipped with a conventional thermionic electron gun and Wehnelt triode. A custom graphite-encircled $\mathrm{LaB}_{6}$ cathode (Applied Physics Technologies) was used for all experiments. The $\mathrm{LaB}_{6}$ is truncated such that the emissive surface is flat and $50 \mu \mathrm{m}$ in diameter. The graphite ensures that emission occurs mainly from the flat $\mathrm{LaB}_{6}$ region and not from the shank, and it seems to also improve photoemission stability compared to bare $\mathrm{LaB}_{6}$ cathodes, though experiments still need to be conducted to explicitly test this. The Tecnai FEMTO is equipped with an optical periscope integrated into the side of the column near the electron gun region (Fig. S1). This periscope provides optical access to the $\mathrm{LaB}_{6}$ cathode such that femtosecond (fs) laser pulses can be used to generate discrete packets of photoelectrons from a room-temperature (i.e., not heated) source. The fs laser used here was a Light Conversion PHAROS (6 W average power; Yb:KGW solidstate laser with regenerative amplifier; $1.03-\mu \mathrm{m}$ fundamental wavelength, 1.2-eV photon energy). Photoelectrons were generated with fourth-harmonic light (4.8-eV photon energy, UV) generated by doubling 515-nm light produced using a Light Conversion HIRO harmonics generator. Laser pulse duration ( $300 \mathrm{fs}$ full-width at half-maximum, FWHM) of the $1.03-\mu \mathrm{m}$ fundamental was measured using a Light Conversion GECO scanning autocorrelator. This duration represents a lower bound for the UV pulse duration due to dispersion introduced during harmonic conversion and delivery to the TEM. 

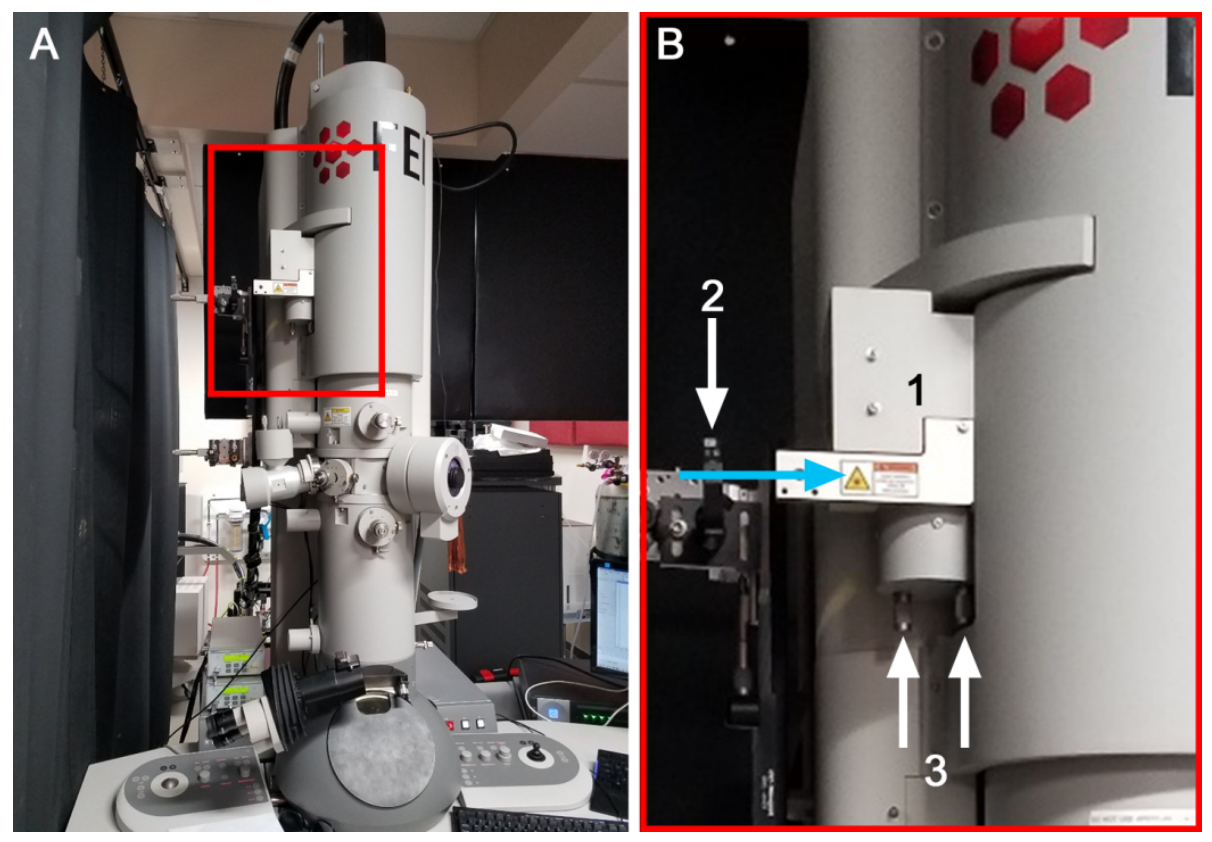

Fig. S1. Thermionic $200 \mathrm{kV}$ TEM modified for laser-driven pulsed operation. (A) Photograph of the modified TEM highlighting (red rectangle) the optical periscope with which the fs UV laser pulses are directed onto the $\mathrm{LaB}_{6}$ electron source. The fs laser system is behind the laser safety curtain to the left outside the frame of the image. (B) Close-up view of the optical periscope highlighted in (A). Denoted in the image are: (1) The optical periscope assembly. (2) An external focusing lens mounted on a 3-axis positioner. (3) Micrometers for manipulating one of the internal mirrors. Direction of the incident UV pulses is indicated by the light-blue arrow.

\section{Controlling Beam Current}

Effort was made to ensure imaging conditions were consistent across all experiments, and especially for directly-compared experiments. Thermionic beam current was controlled primarily using the cathode heat-to value. At times, spot size (i.e., the $\mathrm{C} 1$ condenser-lens setting) was changed slightly to fine-tune the beam current, but this was kept as close to the largest spot size of one as possible in order to ensure consistency and to maintain similarity to the relatively large photoelectron beam spot size used here. Pulsed photoelectron beam current was controlled by varying the fs UV laser pulse energy, which was used to finely control the number of electrons generated per laser pulse (Fig. S2), and via the laser repetition rate ( $f$, pulses per second), which was used to control the number of discrete electron packets generated per unit time. While the overall linear behavior shown in Fig. S2 is typical when operating well below the space-charge (i.e., saturation) limit (1-3), the precise values of the slope and the intercept can be highly variable from one $\mathrm{LaB}_{6}$ cathode to the next, and even from one experiment to the next. Though the factors are likely numerous and highly synergistic, it is reasonable to assume the sources of variability include the changing condition of the cathode surface (e.g., composition, morphology), variation in the laser focusing conditions on the cathode, variation in laser alignment onto the cathode, etc. Additional details specific to Fig. S2 are given in the caption. In light of this, care was taken to ensure each individual experiment, and each set of directlycompared experiments, were self-consistent and consistent, respectively. For example, the photoelectrons per laser pulse were measured and adjusted both before and after every 
experimental run, and the experiment was rejected and repeated if the difference was larger than the random error of the measurement. No reliance was placed on a predetermined quantitative relationship (i.e., a universally applied calibration curve) between the UV laser pulse energy and the measured number of photoelectrons generated per pulse. Details regarding measuring beam currents are provided in the Measuring Beam Current section.
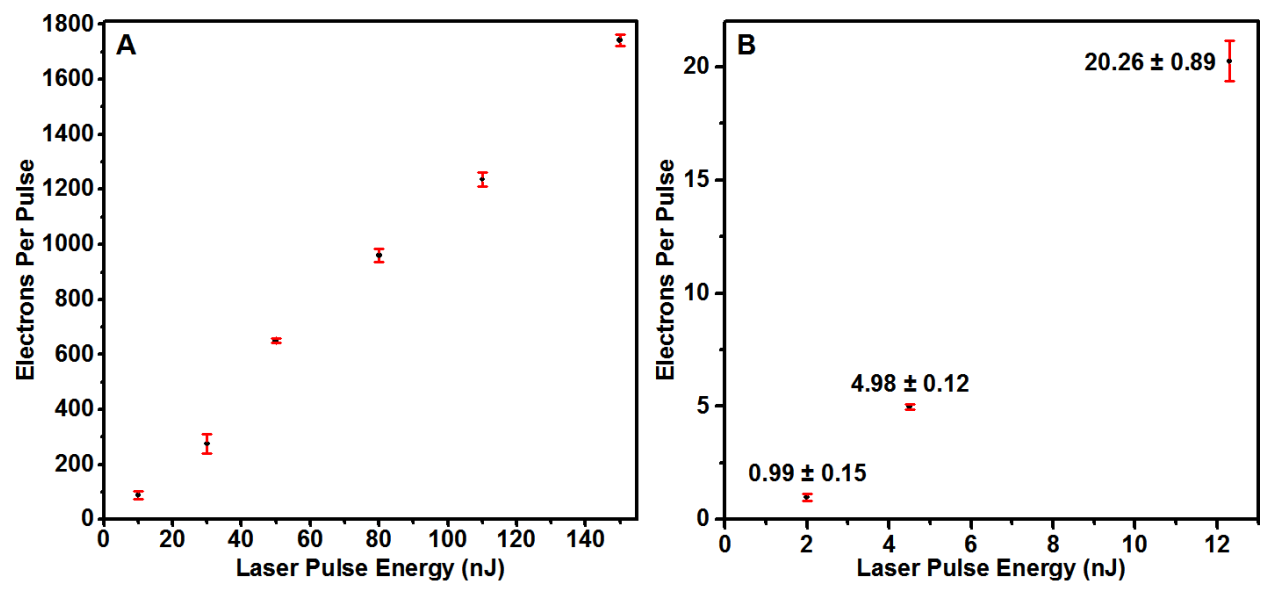

Fig. S2. Photoelectrons per laser pulse generated from the TEM LaB 6 source as a function of pulse energy. (A) Representative behavior of the photoelectrons per pulse for high pulse energies relative to those used to conduct the ultralow-dose-rate experiments. This plot is provided only as a general example of the overall behavior below the onset of saturation. (B) Photoelectrons per pulse at the low pulse energies used for the ultralow-dose-rate experiments reported here. It is important to note that while the data shown in each panel are self-consistent, variation across the data sets is present. For example, in (A), the number of electrons generated per pulse for a laser pulse energy of $10 \mathrm{~nJ}$ was $89 \pm 14$, while in (B), the number was $20 \pm 1$ for a pulse energy of $12.3 \mathrm{~nJ}$. The reason for this is that the data sets were acquired in separate trials. Though the qualitative behaviors will be consistent (e.g., linear response in the relatively low pulse-energy regime), variations in $\mathrm{LaB}_{6}$ photocathode condition, precise laser alignment onto the photocathode, and the precise laser focusing condition onto the photocathode all are expected to cause variation in the absolute photoelectron values. For all experiments, the incident photon energy was $4.8 \mathrm{eV}$ (fourth harmonic of the 1.03- $\mu \mathrm{m}$ fundamental wavelength of the $\mathrm{Yb}: \mathrm{KGW}$ fs laser), and the pulse duration was $300 \mathrm{fs}$ FWHM for the fundamental beam (i.e., lower bound for the UV pulses). The error bars represent one standard error over ten separate measurements.

\section{Experimental Parameters}

Table S1 is a collection of pertinent parameters associated with all experiments presented and discussed in the main text (in addition to a repeat of the experiment shown in Fig. 3; included to demonstrate repeatability). The experiment number corresponds to the figures in the main text; for example, experiment 2.1 is the first experiment associated with Fig. 2, and experiment 4A.1 is the first experiment associated with Fig. 4A. Note that in all cases, the beam area error is less than 1\%. See the section Beam Area Determination for details of these measurements. 


\begin{tabular}{|c|c|c|c|c|c|c|c|c|c|c|c|}
\hline Exp. & $\begin{array}{l}\text { Beam } \\
\text { Type }\end{array}$ & $\begin{array}{c}\text { TBP } \\
(\mu \mathrm{s})\end{array}$ & $\mathbf{E} / \mathbf{P}$ & $\begin{array}{c}\text { E/P } \\
\text { Error }\end{array}$ & $\begin{array}{c}\text { Beam } \\
\text { Current } \\
\text { (fA) }\end{array}$ & $\begin{array}{c}\text { Beam } \\
\text { Current } \\
\text { Error } \\
\text { (fA) } \\
\end{array}$ & $\begin{array}{c}\text { Beam } \\
\text { Area } \\
\left(\mu \mathrm{m}^{2}\right)\end{array}$ & $\begin{array}{c}\text { Dose } \\
\text { Rate } \\
\left(\mathbf{e} \cdot \AA^{-2} \cdot \mathbf{s}^{-1}\right)\end{array}$ & $\begin{array}{l}\text { Acq. } \\
\text { Time } \\
\text { (s) }\end{array}$ & $\begin{array}{l}\text { Total } \\
\text { Time } \\
\text { (hrs) }\end{array}$ & $\begin{array}{c}\text { Final } \\
\Delta I_{110} \\
(\%)\end{array}$ \\
\hline 2.1 & Pulsed & 100 & 1.03 & 0.15 & 1.6 & 0.24 & 132.4 & $7.76 \mathrm{E}-7$ & 90 & 22.4 & -0.81 \\
\hline 2.2 & Therm & $\mathrm{n} / \mathrm{a}$ & $\mathrm{n} / \mathrm{a}$ & $\mathrm{n} / \mathrm{a}$ & 1.6 & 0.22 & 131.5 & 7.77E-7 & 90 & 22.3 & -1.55 \\
\hline 3.1 & Pulsed & 5 & 1.04 & 0.11 & 33.3 & 3.45 & 218.9 & $9.52 \mathrm{E}-6$ & 10 & 1.82 & -0.93 \\
\hline 3.2 & Therm & $\mathrm{n} / \mathrm{a}$ & $\mathrm{n} / \mathrm{a}$ & $\mathrm{n} / \mathrm{a}$ & 32.2 & 3.71 & 218.6 & $9.20 \mathrm{E}-6$ & 10 & 1.89 & -1.66 \\
\hline 3.3 & Pulsed & 5 & 0.99 & 0.12 & 31.7 & 3.72 & 219.4 & $9.04 \mathrm{E}-6$ & 10 & 1.92 & -0.92 \\
\hline 3.4 & Therm & $\mathrm{n} / \mathrm{a}$ & $\mathrm{n} / \mathrm{a}$ & $\mathrm{n} / \mathrm{a}$ & 32 & 3.45 & 220.7 & $9.06 e-6$ & 10 & 1.91 & -1.73 \\
\hline 4A.1 & Pulsed & 5 & 4.96 & 0.16 & 158.7 & 4.97 & 98.7 & $1.00 \mathrm{E}-4$ & 5 & 0.173 & -4.89 \\
\hline 4A.2 & Pulsed & 5 & 5.02 & 0.05 & 160.8 & 1.64 & 98.8 & $1.02 \mathrm{E}-4$ & 5 & 0.171 & -5.60 \\
\hline 4A.3 & Pulsed & 5 & 0.95 & 0.13 & 30.4 & 4.23 & 98.4 & $1.93 \mathrm{E}-5$ & 5 & 0.898 & -0.52 \\
\hline 4A.4 & Pulsed & 5 & 0.89 & 0.14 & 28.5 & 4.56 & 98.7 & $1.81 \mathrm{E}-5$ & 5 & 0.961 & -1.31 \\
\hline 4A.5 & Pulsed & 20 & 0.99 & 0.06 & 7.9 & 0.50 & 98.4 & $5.02 \mathrm{E}-6$ & 5 & 3.46 & -1.53 \\
\hline 4A.6 & Pulsed & 20 & 1.08 & 0.10 & 8.6 & 0.78 & 98.9 & $5.47 \mathrm{E}-6$ & 5 & 3.18 & -3.75 \\
\hline 4A.7 & Pulsed & 20 & 5.04 & 0.05 & 40.3 & 0.37 & 98.7 & $2.55 \mathrm{E}-5$ & 5 & 0.680 & -7.49 \\
\hline 4A.8 & Pulsed & 20 & 4.96 & 0.06 & 39.7 & 0.52 & 98.6 & $2.52 \mathrm{E}-5$ & 5 & 0.690 & -7.70 \\
\hline 4B.1 & Pulsed & 5 & 20.15 & 0.61 & 644.7 & 19.38 & 221.8 & $1.82 \mathrm{E}-4$ & 2 & 0.096 & -9.45 \\
\hline 4B.2 & Pulsed & 5 & 19.71 & 0.56 & 630.6 & 18.07 & 221.1 & $1.78 \mathrm{E}-4$ & 2 & 0.097 & -9.72 \\
\hline 4B.3 & Pulsed & 5 & 19.54 & 1.30 & 625.2 & 41.72 & 220.4 & $1.77 \mathrm{E}-4$ & 2 & 0.098 & -14.79 \\
\hline 4B.4 & Pulsed & 5 & 20.32 & 1.13 & 650.2 & 36.17 & 220.1 & $1.85 \mathrm{E}-4$ & 2 & 0.094 & -11.25 \\
\hline 4B.5 & Pulsed & 5 & 19.53 & 0.75 & 624.8 & 23.99 & 221.3 & $1.76 \mathrm{E}-4$ & 2 & 0.098 & -7.62 \\
\hline 4B.6 & Therm & $\mathrm{n} / \mathrm{a}$ & $\mathrm{n} / \mathrm{a}$ & $\mathrm{n} / \mathrm{a}$ & 627.5 & 16.92 & 220.8 & $1.78 \mathrm{E}-4$ & 2 & 0.098 & -9.86 \\
\hline 4B.7 & Therm & $\mathrm{n} / \mathrm{a}$ & $\mathrm{n} / \mathrm{a}$ & $\mathrm{n} / \mathrm{a}$ & 632.4 & 24.01 & 220.4 & $1.79 \mathrm{E}-4$ & 2 & 0.097 & -6.48 \\
\hline 4B.8 & Therm & $\mathrm{n} / \mathrm{a}$ & $\mathrm{n} / \mathrm{a}$ & $\mathrm{n} / \mathrm{a}$ & 636.9 & 15.99 & 220.9 & $1.80 \mathrm{E}-4$ & 2 & 0.096 & -7.82 \\
\hline 4B.9 & Therm & $\mathrm{n} / \mathrm{a}$ & $\mathrm{n} / \mathrm{a}$ & $\mathrm{n} / \mathrm{a}$ & 652.0 & 19.32 & 221.4 & $1.84 \mathrm{E}-4$ & 2 & 0.094 & -11.59 \\
\hline 4B.10 & Therm & $\mathrm{n} / \mathrm{a}$ & $\mathrm{n} / \mathrm{a}$ & $\mathrm{n} / \mathrm{a}$ & 649.1 & 18.60 & 220.0 & $1.84 \mathrm{E}-4$ & 2 & 0.094 & -10.85 \\
\hline
\end{tabular}

Table S1. Experimental parameters and associated errors for all experiments presented in the main text, as well as one repetition that is not shown in the main text but is reported here to demonstrate repeatability (Exps. 3.3 and 3.4). Exp. = Experiment number, where the first number is the figure number in the main text, and the second number is the specific experiment referred to. Beam Type $=$ pulsed beam $($ Pulsed $)$ or random thermionic beam $($ Therm $)$. TBP $=$ time between electron-packet arrival at the specimen. $\mathrm{E} / \mathrm{P}=$ electrons per packet. Beam Area $=$ size of the electron beam. Acq. Time $=$ camera exposure used to acquire each diffraction pattern. Total Time $=$ the total time required to conduct the fading-curve experiment up to a predetermined total dose. Final $\Delta I_{110}=$ the final average intensity value of the 110 Bragg spots up to the total accumulated dose specific to the experiment.

Photoelectron Beam-Current Stability

Instrument and lab-environment stability were especially critical to the successful execution of all experiments reported here, as the lowest-applied dose rates required over 22 continuous hours of data acquisition. Thus, long-term stability of the electron beam and the 
laboratory room temperature were regularly checked and continuously monitored. If the beam current (pulsed or thermionic) at the conclusion of a data-acquisition run was different from that at the start by more than the measured random error, or if the lab temperature changed by more than $1{ }^{\circ} \mathrm{C}$ during the experiment, the data set was rejected and the experiment was repeated, regardless of the outcome. Lab temperature was continuously monitored at three locations: at the lab door, on the fs laser table, and at the TEM column. Changes of more than $1{ }^{\circ} \mathrm{C}$ at any of these locations at any point during the run resulted in rejection of the entire experiment, again regardless of the outcome. To illustrate the high levels of long-term instrument and lab stability that were reached here, Fig. S3 shows a representative plot of photoelectron counts (measured every 15 minutes by acquiring an image of the condensed beam) as a function of time over a 24hour period. In this data, two standard deviations from the mean corresponds to a range of $\pm 0.3 \%$ from the mean, and a drop in counts of 26 electrons per hour (or $0.0016 \%$ of the mean value) was detected, well below the range of standard deviation arising from random error. See the section Thermal Specimen Drift and Fig. S9 for a discussion and demonstration of the longterm spatial stability of the specimen with respect to drift.

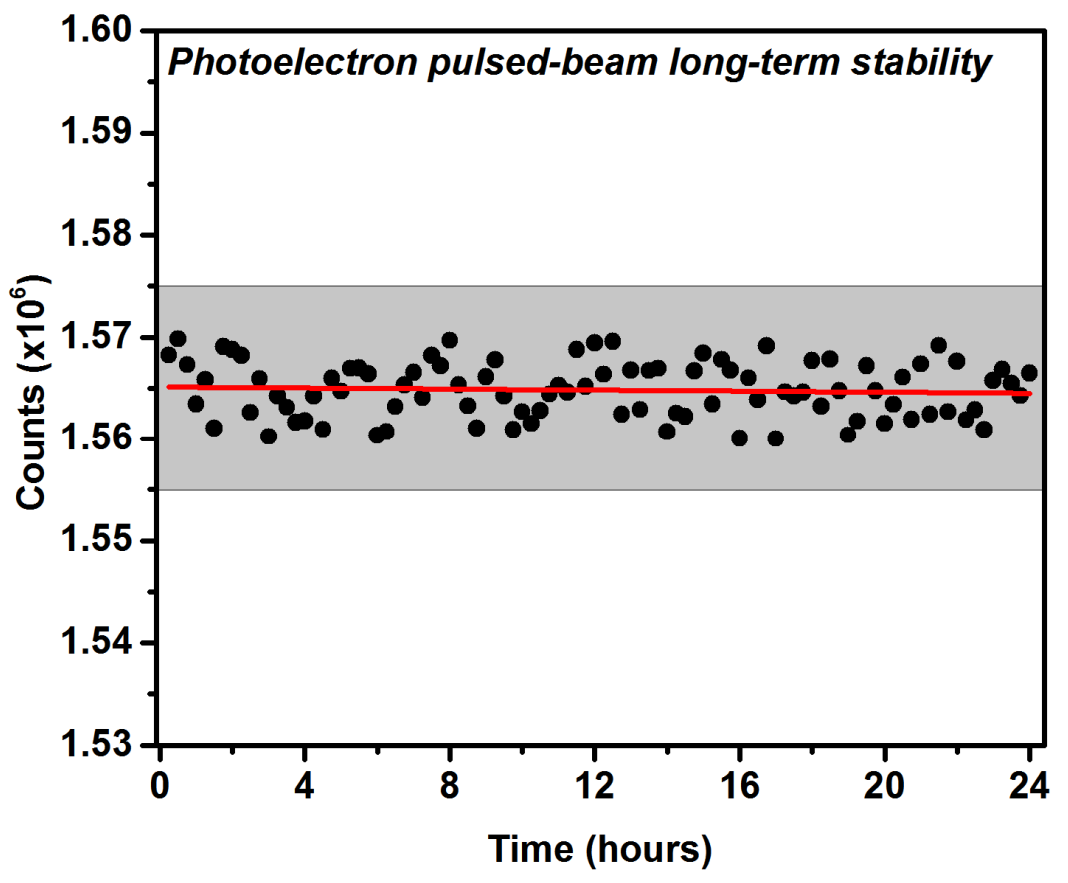

Fig. S3. Representative example of the long-term stability of the pulsed photoelectron beam over a 24-hour period. The data were obtained by summing the counts of an image of the electron source acquired at 15-minute intervals and then converting to the number of electrons incident on the detector. The mean value is $1.565 \pm 0.005 \times 10^{6}$ counts (electrons), where \pm $0.005 \times 10^{6}$ (i.e., $\pm 0.3 \%$ ) is two standard deviations from the mean. The slope of the leastsquares fit line (red) is -25.7 , signifying a drop of 25.7 counts per hour, or approximately $0.0016 \%$ of the mean over 24 hours. To further emphasize the high long-term stability, the grey box denotes a $\pm 0.64 \%$ deviation from the mean. 


\section{Bragg-Spot Fading Curve Method}

A decrease of 110 Bragg-spot intensity as a function of dose and dose rate was used to track and quantify beam damage. An example at high total dose (relative to the experiments reported in the main text) of the type of data generated using this method is shown in Fig. S4. In this case, a hexatriacontane $\left(\mathrm{C}_{36} \mathrm{H}_{74}, \mathrm{C} 36\right)$ microcrystal was irradiated with a $0.57 \mathrm{nA}$ thermionic (random) beam, and a series of diffraction patterns (DPs) were acquired at steadily increasing dose. Progression of the damage manifests as a loss of intensity in the Bragg spots, with the higher-order spots being more sensitive than those generated at lower scattering angles (i.e., larger planar spacings). The average intensity of spots generated from a family of planes is then plotted as a function of dose in order to quantify a damage rate. The dose, $D$, is $D=(I \cdot t) \cdot \mathrm{A}^{-1}$, where $I$ is the beam current $\left(\mathrm{e} \cdot \mathrm{s}^{-1}\right), t$ is the exposure time $(\mathrm{s})$, and $A$ is beam area $\left(\AA^{-2}\right)$. For each experiment described in this work, a single C36 crystal was centered in the field of view, and DPs were acquired at regular intervals up to a predetermined total dose (e.g., $\left.0.063 \mathrm{e} \cdot \AA^{-2}\right)$. The predetermined dose was selected by balancing the need for high stability with the need for a sufficient amount of reliable data for making comparisons and drawing conclusions. Note that while an exponentially-decaying fading curve is shown here and in Fig. 1C, we also observed linearly-decaying behaviors at high total doses. Variation in the functional form of Bragg-spot fading curves at high dose rates has been noted many times in the literature, especially in crystalline paraffins (4-13), and there are likely numerous possible sources $(14,15)$. However, the data displayed in Figs. 2 through 4 of the main text and in Fig. S5 below extend over comparatively small accumulated-dose ranges and, thus, remained within a regime where a linear appearance is expected. While further experiments regarding the origins of different fadingcurve shapes are perhaps merited, such work is well outside the scope of the present study.
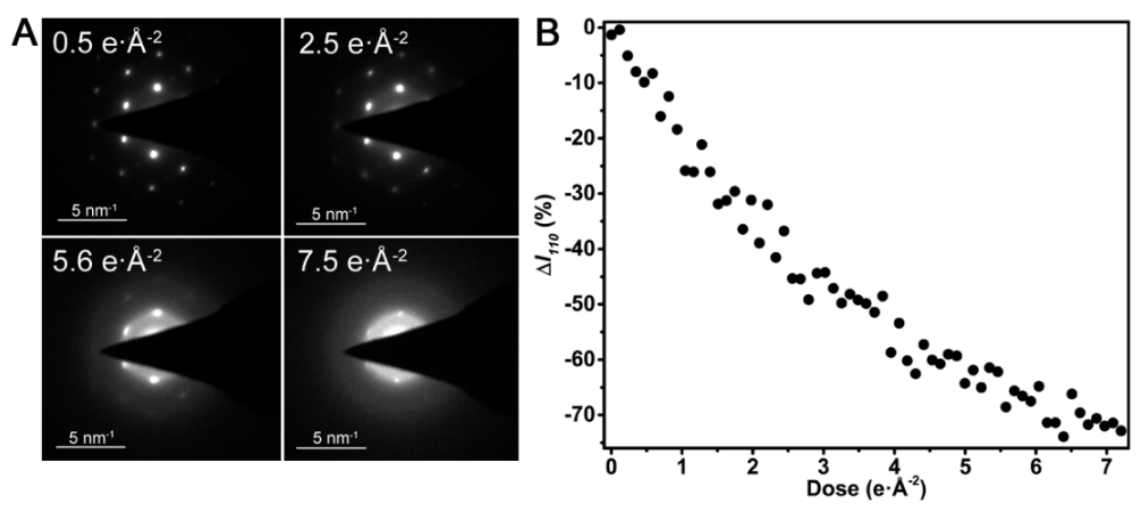

Fig. S4. High-dose example of the Bragg-spot fading-curve method for monitoring beaminduced damage as a function of accumulated dose. (A) Representative diffraction patterns (DPs) from a C36 microcrystal as a function of dose $\left(\mathrm{e} \cdot \AA^{-2}\right)$. (B) Difference in intensity of the 110 Bragg spots as a function of dose relative to the intensities at nominally zero dose. Here, $\Delta I_{110}=\left(I_{110}-I_{0,110}\right) / I_{0}, 110$, where $I_{110}$ is the average 110 Bragg-spot intensity at a non-zero dose, and $I_{0,110}$ is the average intensity at nominally zero dose. The DPs and fading curve shown here are also displayed in Figure 1 in the main text. It is emphasized that the example fading curve shown is for a large total dose relative to the ultra-low dose experiments that were the focus of study. 
For the low-dose experiments that were the subject of study here, fading curves were least-squares fit with a line, and the resulting fit equation was used to calculate the value of $\Delta I_{110}$ at a predetermined dose (e.g., $\left.0.063 \mathrm{e} \cdot \AA^{-2}\right)$, as shown in Fig. S5. Expectation of linearity at these low accumulated doses is further supported by noting that the value of $\Delta I_{110}$ at the predetermined dose was not affected by the overall functional form of the fading curve. This was tested by completing a paired $t$-test on experimental data, with one set displaying a linearly-decaying behavior for a high total dose and the other displaying an exponentially-decaying behavior for a high total dose. The $p$-value on this paired $t$-test was 0.5 , indicating no significant difference between the data generated from a linear vs. an exponential fading-curve behavior at $95 \%$ confidence.

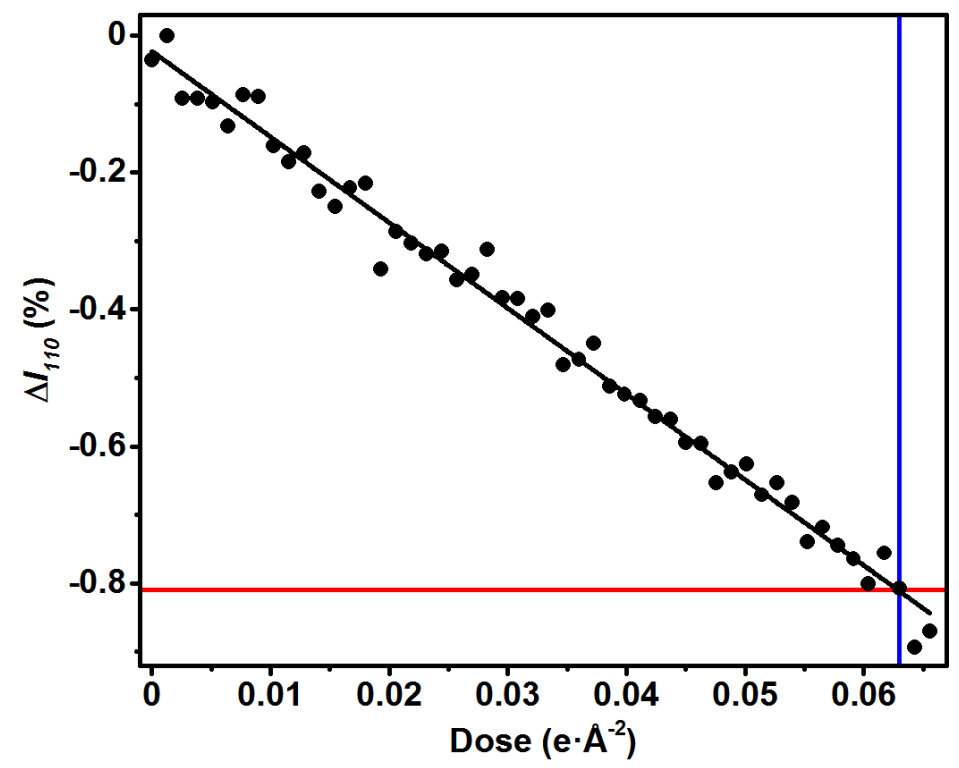

Fig. S5. Method for determining the value of $\Delta I_{110}$ at a predetermined total dose (here, 0.063 e. $\AA^{-2}$ ). The vertical blue line marks the position of a dose of $0.063 \mathrm{e} \cdot \AA^{-2}$, the black line is the linear least-squares fit to the data (black circles), and the horizontal red line marks the position of the value of $\Delta I_{110}$ at the predetermined dose, as per solution to the equation for the linear leastsquares fit.

\section{$\underline{\text { Specimen Preparation }}$}

Hexatriacontane $\left(\mathrm{C}_{36} \mathrm{H}_{74}, \mathrm{C} 36\right)$ microcrystals were used as a model material for several reasons. First, damage due to electron-beam irradiation has been previously studied, and a range of critical doses (i.e., dose producing a Bragg-spot intensity drop of $e^{-1}$ or approximately $37 \%$ ) have been reported (16-18). This provides benchmarks and points of reference for results of both the thermionic- and the pulsed-beam experiments reported here. Second, microcrystals oriented along the [001] crystallographic zone axis and with easily-quantifiable dimensions suitable for study with TEM are readily formed via drop casting from an organic solvent (e.g., decane) onto conductive amorphous carbon grids. In addition, the crystals so formed have a low intrinsic defect density and a nearly monodisperse thickness distribution (see below). Thus, the influence of thickness on measured radiation damage is effectively normalized out for all experiments (19). 
These properties are ideal for identifying multiple crystals suitable for beam-damage studies in order to generate statistics and for precisely determining geometric dimensions. It should be noted that care was exercised in accounting for elastic deformation, strain relaxation, and $n$-beam dynamical scattering in the crystals, as these effects will impact the measured Bragg-spot intensities (16). Specifically here, specimen preparation consisted of drop casting a solution of C36 (Fluka Analytical, 98\% purity) in decane (Acros, 99+\% purity) onto a copper grid with a 12nm-thick amorphous carbon substrate (Electron Microscopy Sciences, Q225-CR4) followed by solvent evaporation in air. A conductive support grid was used in order to reduce the effects of beam-induced specimen charging $(14,20)$. This method produces well-dispersed C36 single microcrystals with a nearly monodisperse thickness of four unit cells, as measured with atomic force microscopy (AFM) and a Bragg-spot intensity-ratio method (described in Thickness of the C36 Specimens).

\section{Measuring Beam Current}

Two methods were used to measure beam current. Method 1 consisted of using a Faraday cup integrated into the specimen holder (Gatan 636.MA double-tilt liquid-nitrogen holder) and connected to a 486 Keithley PicoAmmeter. Method 2 consisted of using the Gatan OneView CMOS camera to determine the counts per unit time and then conversion of this value to a number of electrons per unit time via a known scintillator calibration factor; note the calibration factor for the OneView camera is integrated into the software such that the number of electrons in the image is displayed. While it is reasonable to assume that the beam current measured with Method 1 can be directly related to the beam current used to irradiate the specimen, the geometry of Method 2 is such that the relationship between the beam current at the specimen and the beam current at the detector must be determined. This was accomplished by determining the ratio of the number of electrons per unit time $(N)$ measured with Method 2 (detector; $N_{\text {det. }}$ ) and Method 1 (Faraday cup; $N_{\text {Faraday }}$ ) at several laser-pulse energies (and therefore several beam currents). The results of such an experiment for $N_{\text {det }} / N_{\text {Faraday }}$ are shown in Fig. S6.

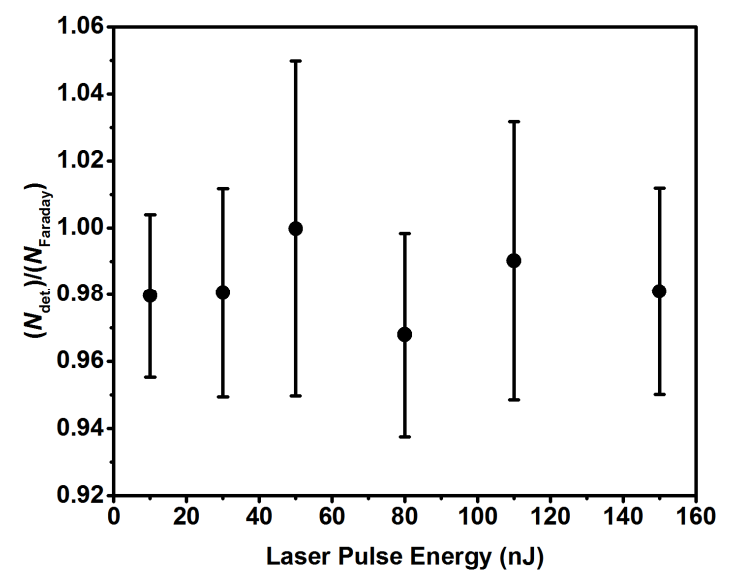

Fig. S6. Number of electrons per unit time measured at the detector $\left(N_{\text {det. }}\right.$, Method 2$)$ relative to the number of electrons measured at the specimen with a Faraday cup for the same amount of time $\left(N_{\text {Faraday }}\right.$, Method 1$)$ for a range of laser pulse energies incident on the TEM photocathode. Error bars represent the standard error over 10 separate measurements. 
Figure S6 demonstrates that, for the same lens settings and projection-system conditions, approximately $98 \%$ of the electrons measured at the specimen plane with the Faraday cup are incident on the detector. A $t$-test at all laser pulse energies tested indicates there is no statistical difference between the two measurement methods to $95 \%$ confidence. Note that the $t$-statistic between the ratio data in Fig. S6 and the value of 1 does not correspond to $p<0.05$, indicating to 95\% confidence that there is no statistical difference between the two forms of measurement. Also note that the overall variability was dominated by random error in the Faraday cup measurements (Method 1); measurements with the Faraday cup had up to 5\% standard error, while those using the detector (Method 2) had a standard error of less than 1\%. The result in Fig. S6 and the statistical equivalency to $95 \%$ confidence indicates it is reasonable to approximate the beam current measured at the detector as the beam current incident on the specimen. Another critical benefit to using Method 2 is the significantly increased sensitivity relative to the Faraday cup and picoammeter; the low random error and high sensitivity relative to the Faraday cup method enabled accurate and precise measurement of the number of electrons per laser pulse down to the lowest of one electron per packet studied here.

Accordingly, method 2 was used to monitor beam-current stability and to determine dose and dose rate for all experiments reported here. This was specifically accomplished by condensing the beam such that it fell entirely on the detector, which was then followed by acquisition of ten images with the same exposure time as the experiment of interest. The total counts for each image was obtained using Digital Micrograph 3. Ultra-Value Analysis was used to identify outliers in counts caused by (for example) X-ray detection. This resulted in rejection of no more than one image in a particular set of 10 (typically all 10 could be used). For each of the usable images, the total electron counts were divided by the image acquisition time to get the electrons per unit time, from which the mean and standard error were determined. The number of electrons per each individual packet could then be determined by noting the laser repetition rate $(f)$, which dictates the number of discrete packets generated per unit time. See Table S1 for a summary of values and associated errors of pertinent experimental parameters.

\section{Beam Area Determination}

Because the electron-beam size may influence the observed damage behaviors (21-23), and because it is a key parameter for calculating dose and dose rate, it must be determined accurately and applied uniformly for direct comparisons to be made. For some experiments, the use of a small beam size (via a small condenser aperture) enabled the beam area to be directly measured. This was done by acquiring an image of the beam at the relevant intensity value and using a Hough transform to calculate the radius. When the beam shape was not circular, an edge-finding algorithm and numerical integration was used. When area could not be determined using a Hough transform or numerical integration (e.g., because portions of the beam fell outside the detector), three additional methods were used (Fig. S7). For one method, an image of the beam was acquired in "M" magnification mode (i.e., standard TEM operation mode; nomenclature specific to FEI instruments) at all intensity values where the entire beam was visible on the detector, and the area was determined as described above. The relationship between intensity and beam size was then plotted and fit with a polynomial. Another method consisted of repeating this process but in "LM" magnification mode (i.e., low magnification mode in which the objective lens is turned off; nomenclature again specific to FEI instruments), enabling a wider range of intensity values to be directly measured. The difference in beam size between the "LM" and the "M" modes was determined, and the average value was used to scale 
the "LM" sizes to the "M" mode. A third method consisted of comparing total counts for a beam imaged entirely on the detector to the counts for a spread beam. By comparing the counts in these two measurements, the proportion of the spread beam falling on the screen can be determined. The area of the spread beam can then be calculated using geometrical arguments despite not being entirely captured on the detector.
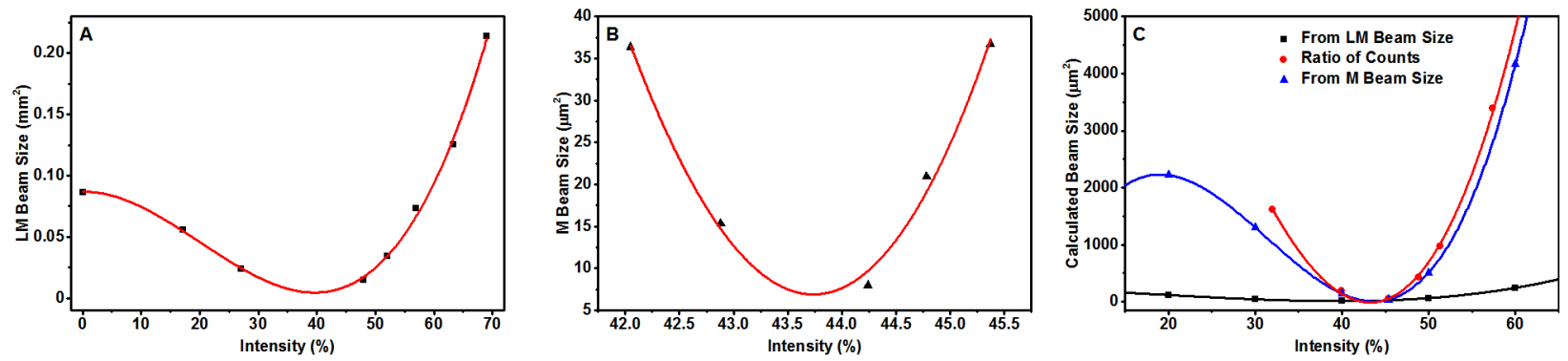

Fig. S7. Methods for determining the electron beam size. (A) Directly measured beam size for a range of intensity values in "LM" magnification mode (black squares). A polynomial fit to the data is shown in red. (B) Directly measured beam size for a range of intensity values in "M" magnification mode (black triangles). A polynomial fit to the data is shown in red. (C) Calculated beam size as a function of intensity value for three different methods over the intensity range of interest: (1) Fit to directly-measured "LM"-mode data (black). (2) Fit to directly-measured "M"-mode data (blue). (3) Geometrical approach based on the ratio of total to measured counts (red).

As can be seen from Fig. S7(C), there is good agreement between the "M" magnification method and the ratio of counts method. The "LM" magnification method was therefore not used. By comparison with similar measurements done with smaller condenser apertures (and therefore under conditions wherein a larger range of intensity values could be directly measured), it was consistently found that both of the retained methods were accurate to within the error of the counts measurement and the Hough transform - about $2 \%$ and $1 \%$, respectively - at the intensity values used in all experiments (i.e., $50 \%$ to $60 \%$ ). Thus, the intensity ratio method for beam-size determination was used for all experiments owing to the ease with which multiple measurements could be quickly performed. This method was typically repeated several times during a given experiment and at least once at the beginning of every experimental run. It is important to note that treating the beam shape as circular was found to be a reasonable approximation when a condenser aperture of $200 \mu \mathrm{m}$ or smaller was used, which was the case for all experiments reported here.

Finally, in addition to controlling for beam size, effort was made to ensure the beam had the same convergence angle in both thermionic and pulsed modes. This was done by ensuring the laser was well-aligned with the center of the $\mathrm{LaB}_{6}$ cathode and that the beam illuminated the entire cathode surface as uniformly as possible. In this way, use of the same lens settings and alignments in the illumination system for both modes of operation resulted in the same beam behavior. This was checked and verified by finding the optimal alignments through standard procedures in both modes and then comparing the resulting lens settings, which were then confirmed to be the same. 


\section{Thickness of the C36 Specimens}

Specimen thickness may affect the damage rates in some organic crystals $(19,24,25)$. Thus, determining the thicknesses of the C36 microcrystals studied here was deemed critical for making direct comparisons between pulsed- and thermionic-beam experiments. However, owing to the extreme beam sensitivity and softness of the microcrystals, it is generally challenging to directly measure thickness either during or immediately before the beam-damage experiment without modifying the structure in a way that could affect the outcome. Measuring crystal thickness after the beam-damage experiment also poses a challenge due to potential mass loss, though this is likely to be minor owing to the low doses and low dose rates studied here. A useful and important feature of C36 microcrystals is that thickness varies by integer multiples of the $c$-axis unit-cell length $(16,26,27)$. Thus, intact crystals will vary in thickness by $n \cdot(c$-axis length), where $n=1,2,3$, etc.

In order to rule out any influence of thickness on observed differences in damage between the pulsed and thermionic beams, a variety of methods were used to determine the distribution of thicknesses of C36 microcrystals formed via the preparation methods described in Specimen Preparation. Thus, the overall likelihood of conducting experiments on crystals of significantly different thickness was established. One method involved measuring the thicknesses of a large number of C36 microcrystals using atomic force microscopy (AFM). The crystals were prepared in the same manner as those used in the beam-damage studies (i.e., same methods as described in Specimen Preparation and on the same type of TEM grid used in the beam-damage experiments). The center thicknesses of 107 individual, isolated (i.e., nonoverlapping) microcrystals were recorded. Of this set, 106 were found to be 19.1-nm thick, while one was found to be 23.8 -nm thick, thus indicating the specimen preparation methods produce a nearly monodisperse thickness distribution.

Other methods employed here made use of the behavior of Bragg-spot intensities with thickness, mainly following work by Dorset (16). One such approach relies on the systematic change in Bragg-spot intensity ratios of the 110 and 200 families of planes with C36 crystal thickness (i.e., number of stacked unit cells along the $c$-axis direction). Though mainly a qualitative measurement, it does allow one to determine if two specific crystals of interest are of approximately the same thickness. For example, for the pulsed and thermionic diffraction patterns shown in Fig. 2 of the main text, the $I_{200} / I_{110}$ ratios were 1.12 and 1.18 , respectively, indicating the thicknesses were nearly the same. Dorset has also shown that an $n$-beam dynamical description is most appropriate for modeling Bragg-spot intensities from C36 crystals $(16,17)$. Indeed, the results of this work demonstrated that forbidden peaks readily appear in C36 crystals that are at least 24-nm thick. As no forbidden peaks were observed in the diffraction patterns analyzed in the beam-damage studies reported here, it is likely that all specimens were less than 24-nm thick, as indicated by the independent AFM measurements.

Dynamical scattering can contribute to allowed Bragg spots as well. Further, because the structure factors of particular reflections for C36 are more affected by $n$-beam scattering than others (e.g., the 220 and 310 compared to the 120 , respectively), and because such scattering is a function of thickness, analyzing intensity ratios of such Bragg spots is another way to determine crystal thickness (16). The results of applying this method here are summarized in Fig. S8. By comparing calculations of Bragg-spot intensity ratios from data reported in (16) to the experimental data reported in Fig. 2 of the main text (for example), it was found that the thicknesses for both $\mathrm{C} 36$ crystals match, within error, the expected values arising from crystals that are 19-nm thick. Taken altogether, the results of the methods used here make it reasonable 
to conclude that the vast majority of C36 microcrystals formed using the described preparation methods were of uniform thickness (i.e., $19 \mathrm{~nm}$ ), and that the likelihood that beam-damage experiments were conducted on microcrystals of differing thicknesses is low.

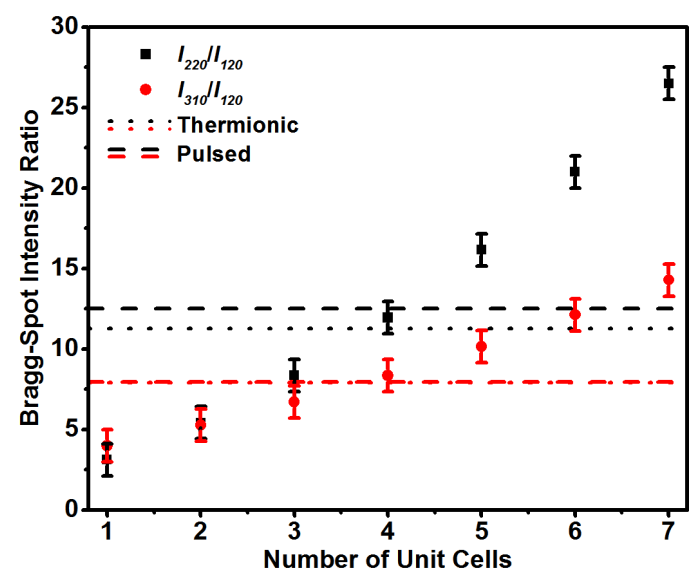

Fig. S8. Measured and calculated Bragg-spot intensity ratios for select reflections from crystalline C36. Individual data points represent the values of $I_{220} / I_{120}$ (black squares) and $I_{310} / I_{120}$ (red circles) as a function of C36 crystal thickness (in number of unit cells) calculated from data reported by Dorset (16). The error bars represent the estimated standard error based on the accuracy of the analysis technique and the estimated accuracy of digitizing the data from the source graph. The horizontal lines correspond to the intensity-ratio values measured here from the diffraction patterns shown in Fig. 2 of the main text, as generated with a thermionic (dotted) and a pulsed (dashed) beam. Note that red horizontal lines corresponding to the $I_{310} / I_{120}$ ratio overlap with one another.

\section{Data Analysis}

After acquisition of all data and recording of all experimental parameters, the data analysis proceeded as follows. First, any shifts in position of the diffraction patterns was corrected using a template-matching plugin in Fiji (28) to calculate the shifts (in pixels in the $x$ and $y$ directions) and a MATLAB code to carry out the correction. This typically resulted in the loss of a few columns and rows of pixels around the frame edges, though no critical Bragg-spot information was lost. Next, the 110 Bragg peaks (the peaks of interest here) for all diffraction patterns in the stack were fit with a 2D Gaussian function, which included background determination. The intensities of the peaks were determined via background removal followed by numerical integration. Because the patterns were analyzed as a stack, the robustness of the automated fitting algorithm was routinely checked. This involved comparison of background values (which did not vary by more than $1 \%$ across all patterns) and comparison of peak fits for the first and last patterns, as well as those at all three quartiles, thus ensuring consistency of background correction and peak fitting across the entire stack. Finally, the intensities were plotted as a function of dose $\left(\mathrm{e} \cdot \AA^{-2}\right)$, and the resulting plots were fit with a linear trend line for the low-dose data. The total change in intensity for each peak was then calculated for a predetermined accumulated dose $\left(e . g ., 0.063 \mathrm{e} \cdot \AA^{-2}\right)$. Note that when peak fitting with a $2 \mathrm{D}$ Gaussian was not ideal, which occasionally occurred for patterns generated with pulsed beams 
due to, for example, a non-circular laser profile on the cathode, an edge-finding algorithm was used to calculate the peak area.

\section{Thermal Specimen Drift}

Owing to relatively long total experiment times for the ultralow-dose studies (see Table $\mathrm{S} 1$ ), determining specimen spatial stability with respect to drift under equilibrated conditions was necessary in order to ensure against potential systematic error arising from, for example, gradual movement of portions of the microcrystal beyond the electron-beam illumination area. Accordingly, the spatial position of the crystal under study was compared before and after the experiment, and minimal to no observable net drift outside of the electron-beam area was observed. Here, net drift refers to specimen drift that results in non-zero lateral motion in the $x y$ plane rather than motion about a single center-of-mass position, as would be expected for a stable and equilibrated system and environment. As suggested by Fig. S3 and discussed in the section Photoelectron Beam-Current Stability, this general observation is a result of the stable equilibrated experimental system and laboratory environment employed here. To further illustrate the typical spatial stability of the specimens with respect to net drift, Fig. S9 displays a bright-field image of a C36 crystal obtained with a $0.8 \mathrm{nA}$ thermionic beam current (much higher than used in the ultralow-dose studies; see Table S1) and a difference image of another image of the same crystal obtained three hours later. As can be seen, only shifts in bend contour position are observed, indicative of slight beam-induced strain relaxation, while no lateral motion is observed at this magnification (the weaker false-colored contrast is noise). This particular experiment was replicated three separate times on three separate C36 crystals with the same results. This illustrates that to within the $12.5 \mathrm{~nm} \cdot \mathrm{pixel}^{-1}$ of this experiment, stability was such that no net specimen drift occurred over the three-hour period. This demonstrates that when the system and laboratory were equilibrated and stable, drift occurs about a single center-of-mass position such that the specimen remained approximately centered within the electron beam, and no change in diffraction signal occurred due to net drift.
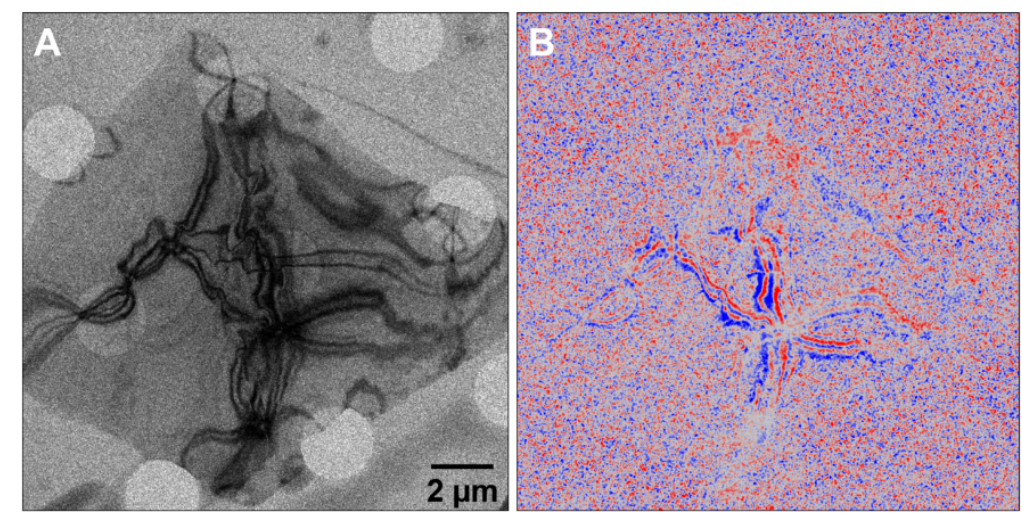

Fig. S9. Demonstration of specimen spatial stability over a three-hour span for the instrument shown in Figure S1. (A) Bright-field image of a single C36 crystal obtained with a $0.8 \mathrm{nA}$ beam current (recall that beam currents of femtoamps were used in the damage studies). (B) Falsecolored difference image formed by subtracting (A) from a bright-field image of the same crystal acquired three hours later. The blue areas represent a loss of counts (i.e., area became darker), and the red areas represent a gain in counts (i.e., area became brighter). 


\section{References}

1. Armstrong MR, Boyden K, Browning ND, Campbell GH, Colvin JD, DeHope WJ, Frank AM, Gibson DJ, Hartemann F, Kim JS, King WE, LaGrange TB, Pyke BJ, Reed BW, Shuttlesworth RM, Stuart BC, Torralva BR (2007) Practical considerations for high spatial and temporal resolution dynamic transmission electron microscopy. Ultramicroscopy 107:356-367.

2. Gahlmann A, Park ST, Zewail AH (2008) Ultrashort electron pulses for diffraction, crystallography and microscopy: Theoretical and experimental resolutions. Phys Chem Chem Phys 10:2894-2909.

3. Plemmons DA, Flannigan DJ (2017) Ultrafast electron microscopy: Instrument response from the single-electron to high bunch-charge regimes. Chem Phys Lett 683:186-192.

4. Kisielowski C, Specht P, Freitag B, Kieft ER, Verhoeven W, van Rens JFM, Mutsaers P, Luiten J, Rozeveld S, Kang J, McKenna AJ, Nickias P, Yancey DF (2019) Discovering hidden material properties of $\mathrm{MgCl}_{2}$ at atomic resolution with structured temporal electron illumination of picosecond time resolution. Adv Funct Mater 29:1807818.

5. Ohno $\mathrm{T}$ (1993) Intensity changes induced by beam damage in electron diffraction from behenic acid multiple monolayers. Ultramicroscopy 48:359-363.

6. Glaeser RM (2016) Chapter two - Specimen behavior in the electron beam. Methods Enzymol 579:19-50.

7. Von Orth H, Fischer EW (1965) Änderungen der Gitterstruktur hochpolymerer Einkristalle durch Bestrahlung im Elektronenmikroskop. Die Makromolekulare Chemie 88:188-214.

8. Grubb DT, Groves GW (1971) Rate of damage of polymer crystals in the electron microscope: Dependence on temperature and beam voltage. Philos Mag 24:815-828.

9. Grubb DT (1974) Radiation damage and electron microscopy of organic polymers. $J$ Mater Sci 9:1715-1736.

10. Salih SM, Cosslett VE (1975) Radiation damage in electron microscopy of organic materials: Effect of low temperatures. J Microsc 105:269-276.

11. Dorset DL, Zemlin F (1985) Structural changes in electron-irradiated paraffin crystals at $<15 \mathrm{~K}$ and their relevance to lattice imaging experiments. Ultramicroscopy 17:229-235. 
12. Dorset DL (1985) Electron crystal structure analysis of small organic molecules. $J$ Electron Microsc Tech 2:89-128.

13. Egerton RF, Rauf I (1999) Dose-rate dependence of electron-induced mass loss from organic specimens. Ultramicroscopy 80:247-254.

14. Egerton RF (2013) Control of radiation damage in the TEM. Ultramicroscopy 127:100108.

15. Egerton RF, Li P, Malac M (2004) Radiation damage in the TEM and SEM. Micron 35:399-409.

16. Dorset D (1980) Electron diffraction intensities from bent molecular organic crystals. Acta Crystallogr Sect A 36:592-600.

17. Dorset D (1976) The interpretation of quasi-kinematical single-crystal electron diffraction intensity data from paraffins. Acta Crystallogr Sect A 32:207-215.

18. Dorset DL, Holland FM, Fryer JR (1984) The "quasi-thermal" mechanism for electron beam damage of n-paraffins. Ultramicroscopy 13:305-310.

19. Fryer JR (1984) Radiation damage in organic crystalline films. Ultramicroscopy 14:227236.

20. Salih SM, Cosslett VE (1974) Reduction in electron-irradiation damage to organic compounds by conducting coatings. Philos Mag 30:225-228.

21. Bullough P, Henderson R (1987) Use of spot-scan procedure for recording low-dose micrographs of beam-sensitive specimens. Ultramicroscopy 21:223-230.

22. Downing KH (1988) Observations of restricted beam-induced specimen motion with small-spot illumination. Ultramicroscopy 24:387-398.

23. Varlot K, Martin JM, Quet C, Kihn Y (1997) Towards sub-nanometer scale EELS analysis of polymers in the TEM. Ultramicroscopy 68:123-133.

24. Salih SM, Cosslett VE (1975) Radiation damage in electron microscopy of organic materials: Effect of low temperatures. J Microsc 105:269-276.

25. Antipenko AP, Bochek GL, Blazhevich SV, Kulibaba VI, Maslov NI, Truten VI, Shramenko BI, Shul'ga NF (1990) The influence of crystal thickness on scattering and radiation of high-energy electrons in oriented crystals. Nucl Instrum Methods Phys Res Sect B 48:291-295.

26. Teare $\mathrm{P}$ (1959) The crystal structure of orthorhombic hexatriacontane, $\mathrm{C}_{36} \mathrm{H}_{74}$. Acta Crystallogr Sect A 12:294-300. 
27. Nyburg SC, Potworowski JA (1973) Prediction of units cells and atomic coordinates for the n-alkanes. Acta Crystallogr Sect B 29:347-352.

28. Schindelin J, Arganda-Carreras I, Frise E, Kaynig V, Longair M, Pietzsch T, Preibisch S, Rueden C, Saalfeld S, Schmid B, Tinevez J-Y, White DJ, Hartenstein V, Eliceiri K, Tomancak P, Cardona A (2012) Fiji: An open-source platform for biological-image analysis. Nat Methods 9:676-682. 